\title{
COVID-19 Severity in Multiple Sclerosis
}

\section{Putting Data Into Context}

\begin{abstract}
Maria Pia Sormani, PhD, Irene Schiavetti, PhD, Luca Carmisciano, MD, Cinzia Cordioli, MD, Massimo Filippi, MD, Marta Radaelli, MD, Paolo Immovilli, MD, Marco Capobianco, MD, Nicola De Rossi, MD, Giampaolo Brichetto, PhD, Eleonora Cocco, MD, Cinzia Scandellari, MD, Paola Cavalla, MD, llaria Pesci, MD, Antonio Zito, MD, Paolo Confalonieri, MD, Girolama Alessandra Marfia, MD, Paola Perini, MD, Matilde Inglese, MD, Maria Trojano, MD, Vincenzo Brescia Morra, PhD, Gioacchino Tedeschi, MD, Giancarlo Comi, MD, Mario Alberto Battaglia, MD, Francesco Patti, MD, and Marco Salvetti, MD, on behalf of the MuSC-19 Study Group
\end{abstract}

Neurol Neuroimmunol Neuroinflamm 2022;9:e1105. doi:10.1212/NXI.0000000000001105

\section{Abstract}

\section{Background and Objectives}

It is unclear how multiple sclerosis (MS) affects the severity of COVID-19. The aim of this study is to compare COVID-19-related outcomes collected in an Italian cohort of patients with MS with the outcomes expected in the age- and sex-matched Italian population.

\section{Methods}

Hospitalization, intensive care unit (ICU) admission, and death after COVID-19 diagnosis of 1,362 patients with MS were compared with the age- and sex-matched Italian population in a retrospective observational case-cohort study with population-based control. The observed vs the expected events were compared in the whole MS cohort and in different subgroups (higher risk: Expanded Disability Status Scale [EDSS] score $>3$ or at least 1 comorbidity, lower risk: EDSS score $\leq 3$ and no comorbidities) by the $\chi^{2}$ test, and the risk excess was quantified by risk ratios (RRs).

\section{Results}

The risk of severe events was about twice the risk in the age- and sex-matched Italian population: $\mathrm{RR}=2.12$ for hospitalization $(p<0.001), \mathrm{RR}=2.19$ for ICU admission $(p<0.001)$, and $\mathrm{RR}=2.43$ for death $(p<0.001)$. The excess of risk was confined to the higher-risk group ( $\mathrm{n}$ $=553)$. In lower-risk patients $(\mathrm{n}=809)$, the rate of events was close to that of the Italian ageand sex-matched population ( $R R=1.12$ for hospitalization, $R R=1.52$ for ICU admission, and $\mathrm{RR}=1.19$ for death). In the lower-risk group, an increased hospitalization risk was detected in patients on anti-CD20 $(\mathrm{RR}=3.03, p=0.005)$, whereas a decrease was detected in patients on interferon ( 0 observed vs 4 expected events, $p=0.04$ ).

\author{
Correspondence \\ Dr. Salvetti \\ marco.salvetti@uniroma1.it
}

\section{MORE ONLINE}

COVID-19 Resources

For the latest articles, invited commentaries, and blogs from physicians around the world NPub.org/COVID19

From the Department of Health Sciences (M.P.S., I.S., L.C.), University of Genova; IRCCS Ospedale Policlinico San Martino (M.P.S., M.I.), Genoa; Centro Sclerosi Multipla ASST Spedali Civili di Brescia (C.C., N.D.R.), Montichiari; Neurology Unit (M.F.), Neurorehabilitation Unit (M.F.), and Neurophysiology Unit (M.F.), IRCCS San Raffaele Scientific Institute, Milan; Neuroimaging Research Unit (M.F.), Division of Neuroscience, IRCCS San Raffaele Scientific Institute, Milan; Vita-Salute San Raffaele University (M.F.), Milan; Department of Neurology and Multiple Sclerosis Center (M.R.), ASST "Papa Giovanni XXIII", Bergamo; Multiple Sclerosis Center (P.I.), Ospedale Guglielmo da Saliceto, Piacenza; Regional Referral Multiple Sclerosis Centre (M.C.), Department of Neurology, University Hospital San Luigi, Orbassano, Torino; AISM Rehabilitation Center (G.B.), Italian MS Society, Genoa; Centro Sclerosi Multipla (E.C.), ATS Sardegna; Dipartimento Scienze Mediche e Sanità Pubblica (E.C.), Università di Cagliari; IRCCS Istituto delle Scienze Neurologiche di Bologna (C.S.), UOSI Riabilitazione Sclerosi Multipla; MS Center (P. Cavalla), Department of Neuroscience, City of Health and Science University Hospital of Turin; Centro SM UOC Neurologia (I. Pesci), Fidenza, AUSL PR; Multiple Sclerosis Research Center (A.Z.), IRCCS Mondino Foundation, Pavia; Multiple Sclerosis Centre (P. Confalonieri), Neuroimmunology Department-“Carlo Besta" Neurological Institute, Milan; Multiple Sclerosis Clinical and Research Unit (G.A.M.), Department of Systems Medicine, Tor Vergata University, Rome; Department of Neurology Multiple Sclerosis Center (P.P.), University of Padua; Department of Neurosciences, Rehabilitation, Ophthalmology, Genetics, Maternal and Child Health (DINOGMI) (M.I.), University of Genoa; Department of Basic Medical Sciences, Neurosciences and Sense Organs (M.T.), University of Bari; Federico II University of Naples (V.B.M.); Department of Advanced Medical and Surgical Sciences (G.T.), University of Campania, Napoli; Università Vita Salute San Raffaele (G.C.), Casa di Cura Privata Del Policlinico, Milan; Research Department (M.A.B.), Italian Multiple Sclerosis Foundation, Genoa; Department of Life Sciences (M.A.B.), University of Siena; Department of Medical and Surgical Sciences and Advanced Technologies (F.P.), GF Ingrassia, University of Catania; Centro Sclerosi Multipla (F.P.), Policlinico Catania, University of Catania; Department of Neuroscience, Mental Health and Sensory Organs (M.S.), Sapienza University of Rome; and Unit of Neurology (M.S.), IRCCS Neuromed, Pozzilli, Isernia, Italy.

Go to Neurology.org/NN for full disclosures. Funding information is provided at the end of the article.

The Article Processing Charge was funded by the authors.

MuSC-19 Study Group coinvestigators are listed in the appendix at the end of the article.

This is an open access article distributed under the terms of the Creative Commons Attribution-NonCommercial-NoDerivatives License 4.0 (CC BY-NC-ND), which permits downloading and sharing the work provided it is properly cited. The work cannot be changed in any way or used commercially without permission from the journal. 


\section{Glossary}

DMT $=$ disease-modifying therapy; EDSS = Expanded Disability Status Scale; ICU = intensive care unit; ISS = Istituto Superiore di Sanità; $\mathbf{M S}=$ multiple sclerosis; $\mathbf{R R}=$ risk ratio; $\mathbf{R T}-\mathbf{P C R}=$ reverse transcriptase-polymerase chain reaction; SARS-CoV-2 = severe acute respiratory syndrome coronavirus 2.

\section{Discussion}

Overall, the MS cohort had a risk of severe events that is twice the risk than the age- and sex-matched Italian population. This excess of risk is mainly explained by the EDSS score and comorbidities, whereas a residual increase of hospitalization risk was observed in patients on anti-CD20 therapies and a decrease in people on interferon.

Several studies have assessed the impact of COVID-19 in patients with multiple sclerosis (MS), unanimously indicating older age, male sex, concomitant comorbidities, and higher disability as risk factors for a more severe disease course. ${ }^{1-4}$ The possible association between immunotherapies and COVID-19 severity was also investigated, mostly indicating an increased risk for patients with MS who are on anti-CD20 therapies or who received methylprednisolone just before the COVID-19 onset $^{1,3,4}$ and suggesting a protective role of interferon. ${ }^{1,4} \mathrm{~A}$ recent metaanalysis of all the published studies on COVID-19 in patients with MS suggested that MS did not significantly increase the mortality rate from COVID- $19,{ }^{5}$ but the authors pointed out that these data should be interpreted with caution as patients with MS are more likely female and younger compared with the general population where age and male sex are risk factors for worse disease outcome. $^{5}$ Therefore, even if all the studies agree that data available so far are overall reassuring, excluding major safety issues, ${ }^{1-4}$ comparisons with external control populations are lacking.

It is unclear whether and how MS biology-apart from treatments-affects the ability to cope with severe acute respiratory syndrome coronavirus 2 (SARS-CoV-2) infection. This is not trivial as immunocompetence, in an immune-mediated disease such as MS, may be reduced. Moreover, SARS-CoV-2 interacts, in a way that is still poorly understood, with the genetic background predisposing to autoimmune diseases (including MS) ${ }^{6}$ and misdirects host immune responses toward autoimmunity as part of COVID-19 pathophysiology. ${ }^{7-9}$ It is therefore plausible that preexisting autoimmunity may exacerbate COVID-19 severity. Therefore, to understand whether patients with MS with COVID-19 are exposed to higher risks than the healthy population, a comparison with an external cohort is needed. The aim of this study is to compare the outcomes collected in an Italian cohort of patients with MS with COVID-19 (within the MuSC-19 project) with the outcomes expected in the age- and sexmatched Italian population, using data provided by the Italian Istituto Superiore di Sanità (ISS).

\section{Methods}

\section{Data Sources: MuSC-19 Study}

Data of patients with MS with suspected or confirmed COVID19 were retrospectively collected at a national level in Italy from February 24, 2020, to February 2, 2021. Details on data collection methods and inclusion criteria were previously reported. ${ }^{1}$ Briefly, we obtained clinician-reported demographic and clinical data on patients with MS with a confirmed or suspected COVID-19 infection from 118 Italian MS centers (eAppendix 2, links.lww. com/NXG/A493). We used a common web-based electronic Case Report Form to collect the data and a unified protocol to analyze them. Demographic, MS history, COVID-19 infection, and follow-up data were collected. For this analysis we included only patients with confirmed COVID-19. To be a confirmed case the patient must have a positive reverse transcriptase-polymerase chain reaction (RT-PCR) nasopharynegal swab.

\section{Data Sources: Italian Population}

We made a specific data request to the ISS, who is the Italian governing body responsible for COVID-19 surveillance in Italy. Data requested (reported in Table 1) were about the percentage of patients who were hospitalized, who accessed intensive care unit (ICU), or who died for each sex and age class $(0-29,30-39,40-49,50-59,60-69,70-79,80-89$, and $>90$ years), among those with a positive RT-PCR during the observation period (February 24, 2020, to February 2, 2021).

\section{Statistical Analysis}

The probability to be hospitalized, to be admitted to ICU, and to die was extracted from ISS data (Table 1) for each patient enrolled in the MuSC-19 data set, according to their age and sex. Then, the expected number of events (e.g., hospitalizations, ICU admissions, or deaths) in the MuSC-19 population and in specific subgroups of patients (detailed below) was estimated by summing up the probabilities for each patient in the group: as an example, if 2 patients have a probability to be hospitalized of 0.5 , the expected number of hospitalizations in this 2-patient group is 1 . The expected proportions of hospitalizations, ICU admissions, and deaths were compared with the observed proportions by a $\chi^{2}$ test, and the relative 
Table 1 COVID-19 Data From the Surveillance Program in Italy

\begin{tabular}{|c|c|c|c|c|c|c|c|c|}
\hline \multirow[b]{2}{*}{ Age } & \multicolumn{4}{|l|}{ Females } & \multicolumn{4}{|l|}{ Males } \\
\hline & Cases ( $\mathrm{N}$ or $\mathrm{n}$ ) & $\operatorname{Hosp}^{a}(\%)$ & ICU (\%) & Death $^{\mathrm{b}}(\%)$ & Cases ( $\mathbf{N}$ or $\mathbf{n}$ ) & $\operatorname{Hosp}^{a}(\%)$ & ICU (\%) & Death $^{\mathrm{b}}(\%)$ \\
\hline $0-29$ & 305,174 & 2.3 & 0.1 & 0.0 & 320,343 & 1.9 & 0.1 & 0.0 \\
\hline $30-39$ & 160,677 & 3.5 & 0.2 & 0.0 & 153,394 & 3.4 & 0.3 & 0.1 \\
\hline $40-49$ & 216,028 & 3.5 & 0.2 & 0.1 & 189,326 & 6.4 & 0.8 & 0.3 \\
\hline $50-59$ & 235,272 & 5.6 & 0.5 & 0.3 & 219,320 & 11.3 & 1.9 & 1.0 \\
\hline $60-69$ & 135,201 & 11.4 & 1.7 & 1.6 & 151,381 & 20.5 & 4.6 & 4.1 \\
\hline $70-79$ & 103,496 & 22.7 & 3.2 & 6.5 & 111,032 & 34.1 & 6.7 & 1.3 \\
\hline $80-89$ & 109,071 & 29.0 & 2.5 & 15.2 & 74,091 & 43.6 & 4.6 & 26.7 \\
\hline$>90$ & 53,545 & 22.7 & 1.3 & 21.8 & 15,884 & 40.6 & 2.6 & 37.6 \\
\hline$U_{n k}^{c}$ & 58 & 0 & 0 & - & 53 & 0 & 0 & 0 \\
\hline Total & $1,318,522$ & & & & $1,234,824$ & & & \\
\hline
\end{tabular}

Abbreviation: $I C U$ = intensive care unit.

admission at hospital.

${ }^{b}$ Data on deaths are relative to the January 29 report.

' Unknown age.

difference expressed as risk ratios (RRs). Binomial 95\% CIs were calculated for the observed proportion of events.

After the comparison of the rate of hospitalization, ICU admission, and death between the MuSC-19 cohort and the ageand sex-matched Italian population was run, we tried to explain the differences observed between patients with MS and the general population by evaluating the role of MS related risk factors, that is, Expanded Disability Status Scale (EDSS) score, comorbidities, and disease-modifying therapy (DMT) exposure, as indicated by previous literature. ${ }^{1-4}$ We focused this additional analysis on hospitalization rates only because the number of observed ICU admissions and deaths was too low to be evaluated in separate subgroups of patients. The same results on observed and expected deaths and ICU admissions are reported in eTable 1 (links.lww.com/NXG/A492).

The specific subgroups of patients were defined according to a cutoff of EDSS score $=3$ and the presence of at least 1 comorbidity. The EDSS score cutoff was chosen based also on the EDSS distribution of the MuSC-19 cohort to have 2 balanced groups. Therefore, the lower-risk group included patients with EDSS score $\leq 3$ and no comorbidities, whereas the higher-risk group included patients with EDSS score $>3$ or at least 1 comorbidity. DMTs were grouped, according to previous literature, as no therapy, interferon therapy, anti-CD20 therapy (rituximab or ocrelizumab), and other DMTs. A $\chi^{2}$ test for heterogeneity was used to compare the RR between groups.

\section{Standard Protocol Approvals, Registrations, and Patient Consents}

The study was approved by the Regional Ethics Committee of Liguria (University of Genoa) (n 130/2020-DB id 10433) and at a national level by Agenzia Italiana del Farmaco.
Written informed consent was obtained from all participants before starting any study procedures.

\section{Data Availability}

MuSC-19 data that support the findings of this study are available on request from the first author (M.P.S). The data are not publicly available due to information that could compromise the privacy of research participants.

\section{Results}

In the MuSC-19 database 1,362 patients with MS had a positive RT-PCR swab for COVID-19 over the observation period and were included in the analysis. The characteristics of the included patients are reported in Table 2. In this cohort, we observed 174 hospitalizations (12.8\%), 22 ICU admissions (1.62\%), and 22 deaths (1.62\%) (not mutually exclusive). The expected number of hospitalizations in an age- and sexmatched cohort extracted from the Italian population was 82 (6.0\%), the expected number of ICU admissions was 10 $(0.73 \%)$, and the expected number of deaths was $9(0.66 \%)$

In Figure 1, the number of observed and expected events is reported. As compared to an age- and sex-matched cohort extracted from the Italian population, the MuSC-19 MS cohort had an excess of hospitalizations $(\mathrm{RR}=2.12,95 \% \mathrm{CI}=$ $1.83-2.44, p<0.001)$, an excess of ICU admissions ( $\mathrm{RR}=$ $2.19,95 \% \mathrm{CI}=1.38-3.30, p=0.007)$, and an excess of deaths $(\mathrm{RR}=2.43,95 \% \mathrm{CI}=1.53-3.66, p=0.007)$.

We tried to explain this excess of risk in 2 steps. First, we checked the MS related risk factors (EDSS score and comorbidities), by splitting the cohort in 2 risk groups, as previously described. The MS lower-risk patients were 809 (60\%), and the MS higher-risk 
Table 2 Characteristics of Patients With MS

\begin{tabular}{|c|c|}
\hline & Overall $(N=1,362)$ \\
\hline Age, mean (SD) & $44.1(12.6)$ \\
\hline Female sex, no. (\%) & $936(68.7)$ \\
\hline \multicolumn{2}{|l|}{ Comorbidities, no. (\%) } \\
\hline Hypertension, no. (\%) & $147(10.8)$ \\
\hline Major depressive disorder, no. (\%) & $49(3.6)$ \\
\hline Hematologic disease, no. (\%) & $38(2.8)$ \\
\hline Diabetes, no. (\%) & $47(3.5)$ \\
\hline Cancer, no. (\%) & $23(1.7)$ \\
\hline Coronary heart disease, no. (\%) & $15(1.1)$ \\
\hline MS duration, median (IQR) & $8.9(3.8-15.9)$ \\
\hline EDSS score, median (IQR) & $2.0(1.0-3.5)$ \\
\hline \multicolumn{2}{|l|}{ MS treatment, no. (\%) } \\
\hline Dimethyl fumarate & $239(17.5)$ \\
\hline Natalizumab & $188(13.8)$ \\
\hline Fingolimod & $166(12.2)$ \\
\hline Ocrelizumab & 149 (10.9) \\
\hline Interferon & $133(9.8)$ \\
\hline Copaxone & $101(7.4)$ \\
\hline Teriflunomide & $79(5.8)$ \\
\hline Cladribine & $27(2.0)$ \\
\hline Rituximab & $27(2.0)$ \\
\hline Azathioprine & $26(1.9)$ \\
\hline Alemtuzumab & $24(1.8)$ \\
\hline Methotrexate & $3(0.2)$ \\
\hline Other & $11(0.8)$ \\
\hline None & 189 (13.9) \\
\hline
\end{tabular}

Abbreviations: EDSS = Expanded Disability Status Scale; IQR = interquartile range; $\mathrm{MS}=$ multiple sclerosis.

patients were 553 (40\%). In the higher-risk group, 119 (22\%) had both EDSS score $>3$ and comorbidities, 150 (27\%) had comorbidities and EDSS score $\leq 3$, and 283 (51\%) had EDSS score $>3$ and no comorbidities. The observed vs expected number of events in these 2 groups is reported in Figure 2. The excess of risk of the MS cohort is mainly confined in the MS higher-risk group: the hospitalization RR was 2.85 (95\% CI = 2.44-3.29, $p<0.001)$ in the higher MS group, whereas it was $1.12(95 \% \mathrm{CI}=0.80-1.52, p=0.44)$ in the lower-risk group (the 2 RRs were significantly heterogeneous, $p<0.001)$. The ICU admission $\mathrm{RR}$ was $2.52(95 \% \mathrm{CI}=1.48-4.00, p<0.001)$ in the MS higher-risk group and $1.52(95 \% \mathrm{CI}=0.49-3.52, p=0.27)$ in the MS lower-risk group (heterogeneity test, $p=0.11$ ). Finally, the death RR was 2.71 (95\% CI $=1.67-4.14, p<0.001)$ in the
MS higher-risk group and $1.19(95 \% \mathrm{CI}=0.14-4.29, p=0.68)$ in the MS lower-risk group (heterogeneity test, $p=0.17$ ).

To try to understand the role of DMTs in explaining the small residual increase of risk in the MS lower-risk group, we split the observed and the expected hospitalization events in 4 groups: untreated patients, patients treated with interferon, patients treated with anti-CD20, and patients treated with other DMTs. In the lower-risk group (Figure 3A), the RRs were significantly heterogeneous among DMT groups $(p=0.048)$ : there was no residual risk in untreated patients $(\mathrm{RR}=1.15,95 \% \mathrm{CI}=$ $0.31-2.92, p=0.78)$ nor in patients treated with other DMTs $(\mathrm{RR}=1.09,95 \% \mathrm{CI}=0.72-1.57, p=0.61)$ as compared to the age- and sex-matched general population; patients with MS treated with interferon had no hospitalization $(\mathrm{RR}=0,95 \% \mathrm{CI}$ $=0-3.7$ ), whereas about 4 were expected, and the difference was statistically significant $(p=0.042)$. Patients treated with anti-CD20 had a significantly higher risk of hospitalization ( $R R$ $=3.03,95 \% \mathrm{CI}=1.30-5.94, p=0.005)$ than the age- and sexmatched general population, showing that the small increase of risk of patients with MS with EDSS score $\leq 3$ and no comorbidities is confined to this class of patients.

In the MS higher-risk group (Figure 3B), the RRs were also significantly heterogeneous among DMTs groups $(p=$ $0.050)$; the $\mathrm{RR}$ was $4.27(95 \% \mathrm{CI}=2.91-6.18, p<0.001)$ for patients under anti-CD20, $3.13(95 \% \mathrm{CI}=2.40-4.04, p<$ $0.001)$ for untreated patients, 2.31 (95\% CI $=1.74-3.04, p<$ $0.001)$ for patients under other DMTs, and 1.80 (95\% CI = $0.66-4.42, p=0.50)$ for patients under interferon.

The number of deaths and ICU admissions according to DMT use in the lower- and in the higher-risk groups is reported in table e-1 (links.lww.com/NXG/A492). In the higher-risk group, the excess of death risk was mainly in the no therapy group $(\mathrm{RR}=3.26,95 \% \mathrm{CI}=1.77-5.37)$ and in the anti-CD20 group $(\mathrm{RR}=5.40,95 \% \mathrm{CI}=1.11-15.25)$, even if the low number of events does not allow to conclude for an heterogeneity of mortality risk according to the DMT group.

\section{Discussion}

Several registries reported the COVID-19 lethality rates of MS cohorts with heterogeneous results, ranging from estimates of $1.6 \%$ in an Italian cohort and $1.7 \%$ in a French cohort ${ }^{4}$ to estimates of $3.6 \%$ in a US cohort. ${ }^{3}$ Explaining these differences is not straightforward and can be linked to the intrinsic limitation of registry data analyses: they are based, in fact, on a voluntary reporting by health care professionals, that may bias collected data toward more severe cases. This may cause an overestimation of clinical severity, with less effect on the internal comparisons among risk factors but challenging external comparisons.

Moreover, comparing the lethality rate of the MS cohorts with the respective national lethality rates in the general population is not meaningful without an adjustment for age and sex. The 
Figure 1 Observed Hospitalizations, ICU Admissions, and Deaths in the MuSC-19 Cohort and Age-Sex-Matched Italian Population

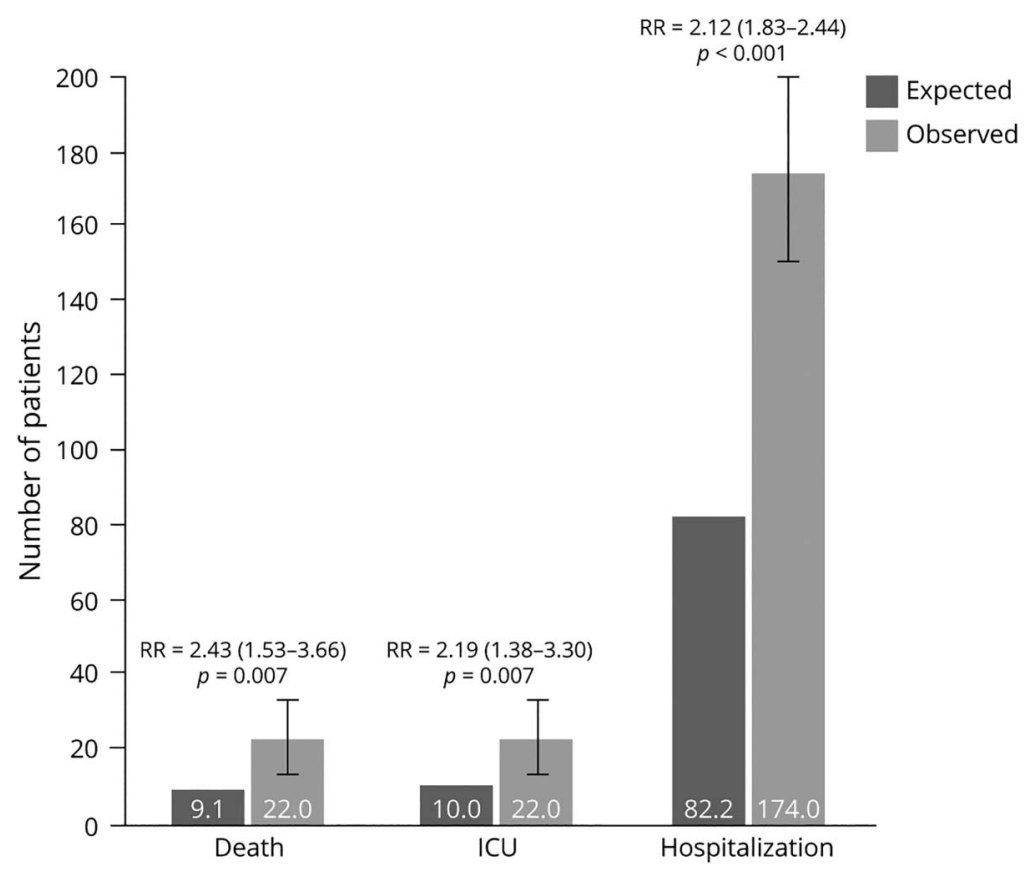

Observed hospitalizations, ICU admissions, and deaths in the MuSC-19 cohort $(n=1,362)$ as compared to the expected number of events in an age and matched cohort from the Italian population. ICU = intensive care unit; RR = risk ratio.

MS population is, in fact, more likely female and younger compared with the general population, and age and male sex are well known risk factors for COVID-19.

This study shows that overall, the patients with MS have a risk of developing a severe COVID-19 that is twice the risk of the age- and sex-matched Italian population. This excess of risk could be in part explained by the abovementioned bias affecting collected data toward more severe cases. However, in patients with MS with a low EDSS score $(\leq 3)$ and no comorbidities, the risk of severe events is very close to the risk of the age- and sex-matched Italian population; in this lower-risk group, only patients under

Figure 2 Observed and Expected Hospitalizations, ICU Admissions, and Deaths in Lower-Risk (A) and Higher-Risk (B) Patients

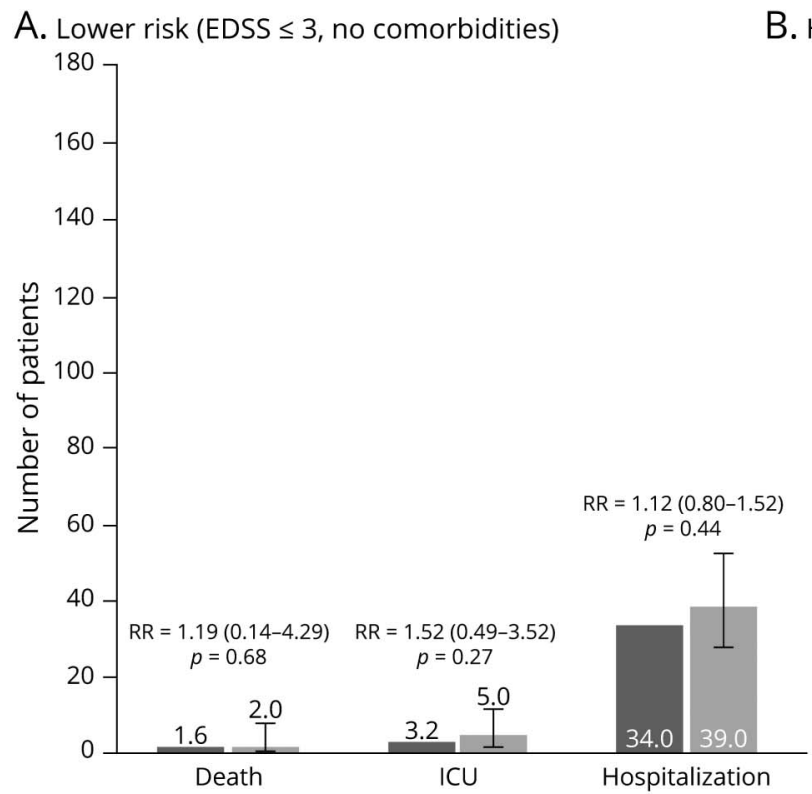

B. Higher risk (EDSS > 3 or comorbidities)

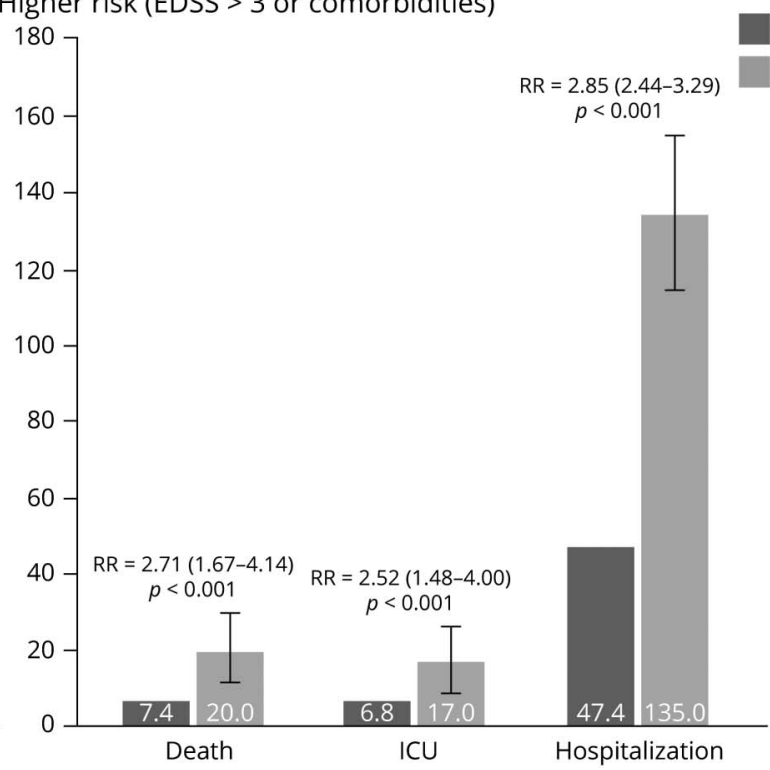

Observed hospitalizations, ICU admissions, and deaths in the lower-risk patients (A; EDSS score $\leq 3$ and no comorbidities, $\mathrm{n}=809$ ) and in the higher-risk patients ( $\mathrm{B}$; EDSS score $>3$ or comorbidities, $\mathrm{n}=553$ ) as compared to the expected number of events in the age and matched cohort from the Italian population. EDSS = Expanded Disability Status Scale; ICU = intensive care unit; RR = risk ratio. 
Figure 3 Observed and Expected Hospitalizations, ICU Admissions, and Deaths According to DMT and Lower-Risk (A) and Higher-Risk (B) Groups

A. Lower risk (EDSS $\leq 3$, no comorbidities)

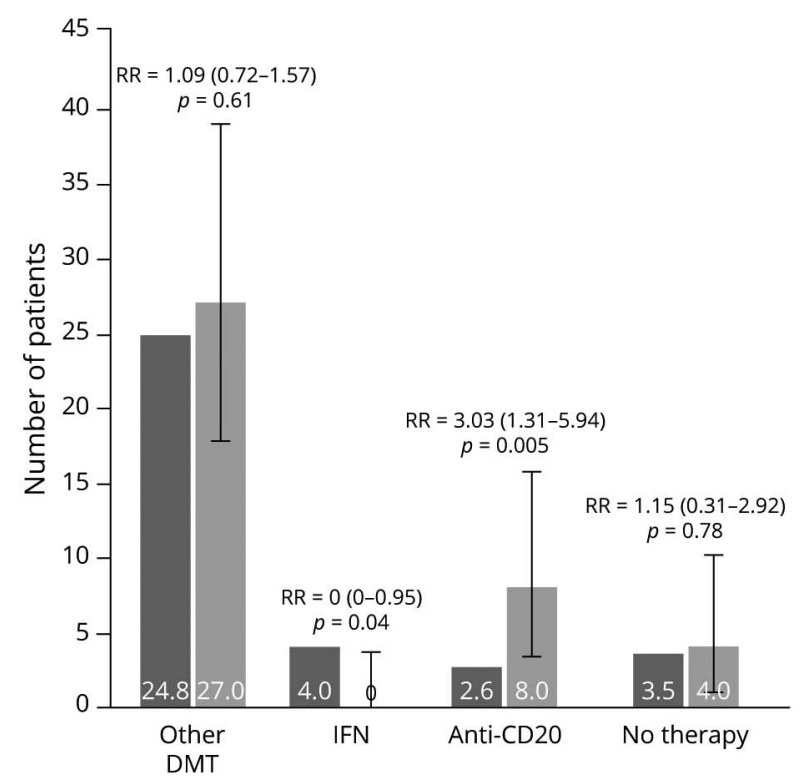

B. Higher risk (EDSS > 3 or comorbidities)

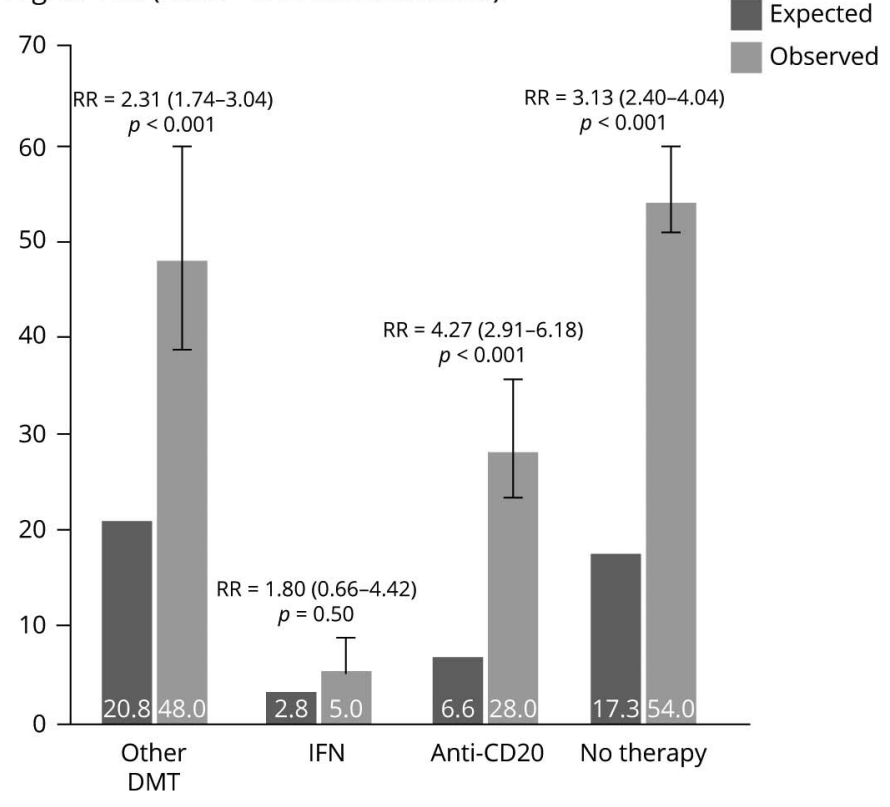

Observed hospitalizations, ICU admissions, and deaths in the lower-risk patients (EDSS score $\leq 3$ and no comorbidities, $\mathrm{n}=809$ ) and in the higher-risk patients (EDSS score > 3 or comorbidities, $n=553$ ) according to the DMT taken as compared to the expected number of hospitalizations in the age- and sex-matched sample from the Italian population. In the interferon group, the RR = 0 because there were no observed events. DMT = disease-modifying therapy; EDSS = Expanded Disability Status Scale; ICU = intensive care unit; IFN = interferon; RR = risk ratio.

anti-CD20 therapy show an increased risk of hospitalization than the age- and sex-matched Italian population. Of interest, the protective role of interferon previously suggested ${ }^{1,3,4}$ is supported here because patients with MS taking interferon show a significantly lower number of hospitalization events than the ageand sex-atched Italian population. In patients with MS, the excess of risk of severe COVID-19 detected is confined to the group of patients with EDSS score $>3$ or with additional comorbidities, were the RR ranges from 1.80 in patients treated with interferon to 4.27 in patients treated with anti-CD20.

The association with disability, and not with the disease itself, suggests that the immunologic defects determining MS do not impair the immunocompetence against SARS-CoV-2 infection. Furthermore, this result is consistent with data from the largest health analytic platforms ${ }^{10}$ where neurologic diseases emerged as factors associated with COVID-19 severe outcome, independently of their immune-mediated pathogenesis. However, we cannot exclude that an increased attention to social distancing ${ }^{11}$ may have counterbalanced the risk of COVID-19 linked to a dysfunctional immune system.

In conclusion, this study shows that in Italy, disability and comorbidities are determinants of an increased risk of severe COVID-19 in patients with MS. Among DMTs, a residual increase of hospitalization is associated with anti-CD20, whereas with interferon, the risk seems to be reduced. These results cannot be generalized because of possibly relevant differences in heritable and nonheritable factors affecting the response to SARS-CoV-2 in different populations. ${ }^{12}$ However, the consistency of the results of previous studies on the impact DMTs on COVID-19 severity in MS, performed in different nations, supports the possibility that our results will be replicated also in other geographic areas and populations.

\section{Acknowledgment}

The MuSC-19 Study Group thanks Roche for donating the web-based platform for data collection, the Department of Informatics, Bioengineering, Robotics, and Systems Engineering, University of Genoa, for its help in installing the platform and Dr. Patrizio Pezzotti for extracting data from the ISS database.

\section{Study Funding}

No targeted funding reported.

\section{Disclosure}

M.P. Sormani reports a grant from Roche to cover data management of the MuSC-19 study; Roche makes ocrelizumab, which is one of the DMTs assessed in this study. Go to Neurology.org/NN for full disclosures.

\section{Publication History}

This manuscript was prepublished in [Sormani, Maria Pia and Schiavetti, Irene and Carmisciano, Luca and Cordioli, Cinzia and Filippi, Massimo and Radaelli, Marta and Immovilli, Paolo and Capobianco, Marco and De Rossi, Nicola and 
Brichetto, Giampaolo and Cocco, Eleonora and Scandellari, Cinzia and Cavalla, Paola and Pesci, Ilaria and Zito, Antonio and Confalonieri, Paolo and Marfia, Girolama Alessandra and Perini, Paola and Inglese, Matilde and Trojano, Maria and Brescia Morra, Vincenzo and Tedeschi, Gioacchino and Comi, Giancarlo and Battaglia, Mario Alberto and Patti, Francesco and Salvetti, Marco and Study Group, MuSC-19, COVID-19 Severity in Multiple Sclerosis: Putting Data Into Context. Available at SSRN: ssrn.com/abstract=3884934]. Received by Neurology: Neuroimmunology \& Neuroinflammation July 15, 2021. Accepted in final form September 15, 2021.

\section{Appendix 1 Authors}

\begin{tabular}{|c|c|c|}
\hline Name & Location & Contribution \\
\hline $\begin{array}{l}\text { Maria Pia } \\
\text { Sormani, PhD }\end{array}$ & $\begin{array}{l}\text { Department of Health } \\
\text { Sciences, University of } \\
\text { Genova, Italy; IRCCS } \\
\text { Ospedale Policlinico San } \\
\text { Martino, Genoa, Italy }\end{array}$ & $\begin{array}{l}\text { Drafting/revision of the } \\
\text { manuscript for content, } \\
\text { including medical writing for } \\
\text { content; study concept or } \\
\text { design; and analysis or } \\
\text { interpretation of data }\end{array}$ \\
\hline $\begin{array}{l}\text { Irene } \\
\text { Schiavetti, } \\
\text { PhD }\end{array}$ & $\begin{array}{l}\text { Department of Health } \\
\text { Sciences, University of } \\
\text { Genova, Italy }\end{array}$ & $\begin{array}{l}\text { Drafting/revision of the } \\
\text { manuscript for content, } \\
\text { including medical writing for } \\
\text { content; major role in the } \\
\text { acquisition of data; study } \\
\text { concept or design; and } \\
\text { analysis or interpretation of } \\
\text { data }\end{array}$ \\
\hline $\begin{array}{l}\text { Luca } \\
\text { Carmisciano, } \\
\text { MD }\end{array}$ & $\begin{array}{l}\text { Department of Health } \\
\text { Sciences, University of } \\
\text { Genova, Italy }\end{array}$ & $\begin{array}{l}\text { Drafting/revision of the } \\
\text { manuscript for content, } \\
\text { including medical writing for } \\
\text { content; study concept or } \\
\text { design; and analysis or } \\
\text { interpretation of data }\end{array}$ \\
\hline $\begin{array}{l}\text { Cinzia } \\
\text { Cordioli, MD }\end{array}$ & $\begin{array}{l}\text { Centro Sclerosi Multipla } \\
\text { ASST Spedali Civili di Brescia, } \\
\text { Montichiari, Italy }\end{array}$ & $\begin{array}{l}\text { Drafting/revision of the } \\
\text { manuscript for content, } \\
\text { including medical writing for } \\
\text { content, and major role in } \\
\text { the acquisition of data }\end{array}$ \\
\hline $\begin{array}{l}\text { Massimo } \\
\text { Filippi, MD }\end{array}$ & $\begin{array}{l}\text { Neurology Unit, IRCCS San } \\
\text { Raffaele Scientific Institute, } \\
\text { Milan, Italy; } \\
\text { Neurorehabilitation Unit, } \\
\text { IRCCS San Raffaele Scientific } \\
\text { Institute, Milan, Italy; } \\
\text { Neurophysiology Unit, IRCCS } \\
\text { San Raffaele Scientific } \\
\text { Institute, Milan, Italy; } \\
\text { Neuroimaging Research Unit, } \\
\text { Division of Neuroscience, } \\
\text { IRCCS San Raffaele Scientific } \\
\text { Institute, Milan, Italy; Vita- } \\
\text { Salute San Raffaele University, } \\
\text { Milan, Italy }\end{array}$ & $\begin{array}{l}\text { Drafting/revision of the } \\
\text { manuscript for content, } \\
\text { including medical writing for } \\
\text { content, and major role in } \\
\text { the acquisition of data }\end{array}$ \\
\hline $\begin{array}{l}\text { Marta } \\
\text { Radaelli, MD }\end{array}$ & $\begin{array}{l}\text { Department of Neurology } \\
\text { and Multiple Sclerosis } \\
\text { Center, ASST Papa Giovanni } \\
\text { XXIII, Bergamo, Italy }\end{array}$ & $\begin{array}{l}\text { Drafting/revision of the } \\
\text { manuscript for content, } \\
\text { including medical writing for } \\
\text { content, and major role in } \\
\text { the acquisition of data }\end{array}$ \\
\hline $\begin{array}{l}\text { Paolo } \\
\text { Immovilli, } \\
\text { MD }\end{array}$ & $\begin{array}{l}\text { Multiple Sclerosis Center, } \\
\text { Ospedale Guglielmo da } \\
\text { Saliceto, Piacenza, Italy }\end{array}$ & $\begin{array}{l}\text { Drafting/revision of the } \\
\text { manuscript for content, } \\
\text { including medical writing for } \\
\text { content, and major role in } \\
\text { the acquisition of data }\end{array}$ \\
\hline
\end{tabular}

Appendix 1 (continued)

\begin{tabular}{|c|c|c|}
\hline Name & Location & Contribution \\
\hline $\begin{array}{l}\text { Marco } \\
\text { Capobianco, } \\
\text { MD }\end{array}$ & $\begin{array}{l}\text { Regional Referral Multiple } \\
\text { Sclerosis Centre, Dept. of } \\
\text { Neurology, University } \\
\text { Hospital San Luigi, } \\
\text { Orbassano, Torino, Italy }\end{array}$ & $\begin{array}{l}\text { Drafting/revision of the } \\
\text { manuscript for content, } \\
\text { including medical writing for } \\
\text { content, and major role in } \\
\text { the acquisition of data }\end{array}$ \\
\hline $\begin{array}{l}\text { Nicola De } \\
\text { Rossi, MD }\end{array}$ & $\begin{array}{l}\text { Centro Sclerosi Multipla } \\
\text { ASST Spedali Civili di Brescia, } \\
\text { Montichiari, Italy }\end{array}$ & $\begin{array}{l}\text { Drafting/revision of the } \\
\text { manuscript for content, } \\
\text { including medical writing for } \\
\text { content, and major role in } \\
\text { the acquisition of data }\end{array}$ \\
\hline $\begin{array}{l}\text { Giampaolo } \\
\text { Brichetto, } \\
\text { PhD }\end{array}$ & $\begin{array}{l}\text { AISM Rehabilitation Center, } \\
\text { Italian MS Society, Genoa, } \\
\text { Italy }\end{array}$ & $\begin{array}{l}\text { Drafting/revision of the } \\
\text { manuscript for content, } \\
\text { including medical writing for } \\
\text { content, and major role in } \\
\text { the acquisition of data }\end{array}$ \\
\hline $\begin{array}{l}\text { Eleonora } \\
\text { Cocco, MD }\end{array}$ & $\begin{array}{l}\text { Centro Sclerosi Multipla, ATS } \\
\text { Sardegna; Dipartimento } \\
\text { Scienze Mediche e Sanità } \\
\text { Pubblica, Università di } \\
\text { Cagliari }\end{array}$ & $\begin{array}{l}\text { Drafting/revision of the } \\
\text { manuscript for content, } \\
\text { including medical writing for } \\
\text { content, and major role in } \\
\text { the acquisition of data }\end{array}$ \\
\hline $\begin{array}{l}\text { Cinzia } \\
\text { Scandellari, } \\
\text { MD }\end{array}$ & $\begin{array}{l}\text { IRCCS Istituto delle Scienze } \\
\text { Neurologiche di Bologna, } \\
\text { UOSI Riabilitazione Sclerosi } \\
\text { Multipla }\end{array}$ & $\begin{array}{l}\text { Drafting/revision of the } \\
\text { manuscript for content, } \\
\text { including medical writing for } \\
\text { content, and major role in } \\
\text { the acquisition of data }\end{array}$ \\
\hline $\begin{array}{l}\text { Paola } \\
\text { Cavalla, MD }\end{array}$ & $\begin{array}{l}\text { MS Center, Department of } \\
\text { Neuroscience, City of Health } \\
\text { and Science University } \\
\text { Hospital of Turin, Italy }\end{array}$ & $\begin{array}{l}\text { Drafting/revision of the } \\
\text { manuscript for content, } \\
\text { including medical writing for } \\
\text { content, and major role in } \\
\text { the acquisition of data }\end{array}$ \\
\hline $\begin{array}{l}\text { Ilaria Pesci, } \\
\text { MD }\end{array}$ & $\begin{array}{l}\text { Centro SM UOC Neurologia, } \\
\text { Fidenza, AUSL PR }\end{array}$ & $\begin{array}{l}\text { Drafting/revision of the } \\
\text { manuscript for content, } \\
\text { including medical writing for } \\
\text { content, and major role in } \\
\text { the acquisition of data }\end{array}$ \\
\hline $\begin{array}{l}\text { Antonio Zito, } \\
\text { MD }\end{array}$ & $\begin{array}{l}\text { Multiple Sclerosis Research } \\
\text { Center, IRCCS Mondino } \\
\text { Foundation, Pavia }\end{array}$ & $\begin{array}{l}\text { Drafting/revision of the } \\
\text { manuscript for content, } \\
\text { including medical writing for } \\
\text { content, and major role in } \\
\text { the acquisition of data }\end{array}$ \\
\hline $\begin{array}{l}\text { Paolo } \\
\text { Confalonieri, } \\
\text { MD }\end{array}$ & $\begin{array}{l}\text { Multiple Sclerosis Centre, } \\
\text { Neuroimmunology } \\
\text { Department-“Carlo Besta" } \\
\text { Neurological Institute, } \\
\text { Milan, Italy }\end{array}$ & $\begin{array}{l}\text { Drafting/revision of the } \\
\text { manuscript for content, } \\
\text { including medical writing for } \\
\text { content, and major role in } \\
\text { the acquisition of data }\end{array}$ \\
\hline $\begin{array}{l}\text { Girolama } \\
\text { Alessandra } \\
\text { Marfia, MD }\end{array}$ & $\begin{array}{l}\text { Multiple Sclerosis Clinical } \\
\text { and Research Unit, } \\
\text { Department of Systems } \\
\text { Medicine, Tor Vergata } \\
\text { University, Rome, Italy }\end{array}$ & $\begin{array}{l}\text { Drafting/revision of the } \\
\text { manuscript for content, } \\
\text { including medical writing for } \\
\text { content, and major role in } \\
\text { the acquisition of data }\end{array}$ \\
\hline $\begin{array}{l}\text { Paola Perini, } \\
\text { MD }\end{array}$ & $\begin{array}{l}\text { Department of Neurology } \\
\text { Multiple Sclerosis Center, } \\
\text { University of Padua, Italy }\end{array}$ & $\begin{array}{l}\text { Drafting/revision of the } \\
\text { manuscript for content, } \\
\text { including medical writing for } \\
\text { content, and major role in } \\
\text { the acquisition of data }\end{array}$ \\
\hline $\begin{array}{l}\text { Matilde } \\
\text { Inglese, MD }\end{array}$ & $\begin{array}{l}\text { Department of } \\
\text { Neurosciences, } \\
\text { Rehabilitation, } \\
\text { Ophthalmology, Genetics, } \\
\text { Maternal and Child Health } \\
\text { (DINOGMI), University of } \\
\text { Genoa, Italy; IRCCS } \\
\text { Ospedale Policlinico San } \\
\text { Martino, Genoa, Italy }\end{array}$ & $\begin{array}{l}\text { Drafting/revision of the } \\
\text { manuscript for content, } \\
\text { including medical writing for } \\
\text { content, and major role in } \\
\text { the acquisition of data }\end{array}$ \\
\hline
\end{tabular}


Appendix 1 (continued)

\begin{tabular}{|c|c|c|}
\hline Name & Location & Contribution \\
\hline $\begin{array}{l}\text { Maria } \\
\text { Trojano, MD }\end{array}$ & $\begin{array}{l}\text { Department of Basic } \\
\text { Medical Sciences, } \\
\text { Neurosciences and Sense } \\
\text { Organs, University of Bari, } \\
\text { Italy }\end{array}$ & $\begin{array}{l}\text { Drafting/revision of the } \\
\text { manuscript for content, } \\
\text { including medical writing for } \\
\text { content, and major role in } \\
\text { the acquisition of data }\end{array}$ \\
\hline $\begin{array}{l}\text { Vincenzo } \\
\text { Brescia } \\
\text { Morra, PhD }\end{array}$ & $\begin{array}{l}\text { Federico II University of } \\
\text { Naples, Italy }\end{array}$ & $\begin{array}{l}\text { Drafting/revision of the } \\
\text { manuscript for content, } \\
\text { including medical writing for } \\
\text { content, and major role in } \\
\text { the acquisition of data }\end{array}$ \\
\hline $\begin{array}{l}\text { Gioacchino } \\
\text { Tedeschi, MD }\end{array}$ & $\begin{array}{l}\text { Department of Advanced } \\
\text { Medical and Surgical } \\
\text { Sciences, University of } \\
\text { Campania, Napoli, Italy }\end{array}$ & $\begin{array}{l}\text { Drafting/revision of the } \\
\text { manuscript for content, } \\
\text { including medical writing for } \\
\text { content, and major role in } \\
\text { the acquisition of data }\end{array}$ \\
\hline $\begin{array}{l}\text { Giancarlo } \\
\text { Comi, MD }\end{array}$ & $\begin{array}{l}\text { Università Vita Salute San } \\
\text { Raffaele, Casa di Cura } \\
\text { Privata del Policlinico, Milan, } \\
\text { Italy }\end{array}$ & $\begin{array}{l}\text { Drafting/revision of the } \\
\text { manuscript for content, } \\
\text { including medical writing for } \\
\text { content, and major role in } \\
\text { the acquisition of data }\end{array}$ \\
\hline $\begin{array}{l}\text { Mario } \\
\text { Alberto } \\
\text { Battaglia, MD }\end{array}$ & $\begin{array}{l}\text { Research Department, } \\
\text { Italian Multiple Sclerosis } \\
\text { Foundation, Genoa, Italy; } \\
\text { Department of Life Sciences, } \\
\text { University of Siena, Italy }\end{array}$ & $\begin{array}{l}\text { Drafting/revision of the } \\
\text { manuscript for content, } \\
\text { including medical writing for } \\
\text { content, and major role in } \\
\text { the acquisition of data }\end{array}$ \\
\hline $\begin{array}{l}\text { Francesco } \\
\text { Patti, MD }\end{array}$ & $\begin{array}{l}\text { Department of Medical and } \\
\text { Surgical Sciences and } \\
\text { Advanced Technologies, GF } \\
\text { Ingrassia, University of } \\
\text { Catania; Centro Sclerosi } \\
\text { Multipla, Policlinico Catania, } \\
\text { University of Catania }\end{array}$ & $\begin{array}{l}\text { Drafting/revision of the } \\
\text { manuscript for content, } \\
\text { including medical writing for } \\
\text { content, and major role in } \\
\text { the acquisition of data }\end{array}$ \\
\hline $\begin{array}{l}\text { Marco } \\
\text { Salvetti, MD }\end{array}$ & $\begin{array}{l}\text { Department of } \\
\text { Neuroscience, Mental } \\
\text { Health and Sensory Organs, } \\
\text { Sapienza University of } \\
\text { Rome, Italy; Unit of Neurology, } \\
\text { IRCCS Neuromed, Pozzilli, } \\
\text { Isernia, Italy }\end{array}$ & $\begin{array}{l}\text { Drafting/revision of the } \\
\text { manuscript for content, } \\
\text { including medical writing for } \\
\text { content, and major role in } \\
\text { the acquisition of data }\end{array}$ \\
\hline
\end{tabular}

Appendix 2 Coinvestigators

\begin{tabular}{|c|c|c|c|}
\hline Name & Location & Role & Contribution \\
\hline $\begin{array}{l}\text { Gianmarco } \\
\text { Abbadessa }\end{array}$ & $\begin{array}{l}\text { Department of Advanced } \\
\text { Medical and Surgical } \\
\text { Sciences, University of } \\
\text { Campania Luigi } \\
\text { Vanvitelli, } 80138 \text { Naples, } \\
\text { Italy; }\end{array}$ & $\begin{array}{l}\text { Site } \\
\text { Investigator }\end{array}$ & $\begin{array}{l}\text { Data } \\
\text { collection }\end{array}$ \\
\hline $\begin{array}{l}\text { Umberto } \\
\text { Aguglia }\end{array}$ & $\begin{array}{l}\text { Department of medical } \\
\text { and surgical sciences, } \\
\text { Magna Graecia } \\
\text { University Catanzaro }\end{array}$ & $\begin{array}{l}\text { Site } \\
\text { Investigator }\end{array}$ & $\begin{array}{l}\text { Data } \\
\text { collection }\end{array}$ \\
\hline Lia Allegorico & $\begin{array}{l}\text { Multiple Sclerosis Centre } \\
\text { A. Cardarelli Hospital, } \\
\text { Naples, Italy }\end{array}$ & $\begin{array}{l}\text { Site } \\
\text { Investigator }\end{array}$ & $\begin{array}{l}\text { Data } \\
\text { collection }\end{array}$ \\
\hline $\begin{array}{l}\text { Beatrice Maria } \\
\text { Allegri Rossi }\end{array}$ & Centro SM Fidenza (PR) & $\begin{array}{l}\text { Site } \\
\text { Investigator }\end{array}$ & $\begin{array}{l}\text { Data } \\
\text { collection }\end{array}$ \\
\hline
\end{tabular}

Appendix 2 (continued)

\begin{tabular}{|c|c|c|c|}
\hline Name & Location & Role & Contribution \\
\hline $\begin{array}{l}\text { Maria Pia } \\
\text { Amato }\end{array}$ & $\begin{array}{l}\text { Università degli Studi di } \\
\text { Firenze, Dipartimento } \\
\text { NEUROFARBA, Firenze - } \\
\text { IRCCS Fondazione Don } \\
\text { Carlo Gnocchi, Firenze }\end{array}$ & $\begin{array}{l}\text { Site } \\
\text { Investigator }\end{array}$ & $\begin{array}{l}\text { Data } \\
\text { collection }\end{array}$ \\
\hline $\begin{array}{l}\text { Pietro } \\
\text { Annovazzi }\end{array}$ & $\begin{array}{l}\text { Centro Sclerosi Multipla } \\
\text { Ospedale di Gallarate, } \\
\text { ASST della Valle Olona }\end{array}$ & $\begin{array}{l}\text { Site } \\
\text { Investigator }\end{array}$ & $\begin{array}{l}\text { Data } \\
\text { collection }\end{array}$ \\
\hline Carlo Antozzi & $\begin{array}{l}\text { Centro Sclerosi Multipla, } \\
\text { U.O Neurologia IV, } \\
\text { Fondazione IRCCS } \\
\text { Istituto Neurologico } \\
\text { "Carlo Besta", Milano }\end{array}$ & $\begin{array}{l}\text { Site } \\
\text { Investigator }\end{array}$ & $\begin{array}{l}\text { Data } \\
\text { collection }\end{array}$ \\
\hline $\begin{array}{l}\text { Lucia } \\
\text { Appendino }\end{array}$ & $\begin{array}{l}\text { SC Neurologia1 Ospedale } \\
\text { Maria Vittoria- Torino }\end{array}$ & $\begin{array}{l}\text { Site } \\
\text { Investigator }\end{array}$ & $\begin{array}{l}\text { Data } \\
\text { collection }\end{array}$ \\
\hline $\begin{array}{l}\text { Sebastiano } \\
\text { Arena }\end{array}$ & $\begin{array}{l}\text { Dipartimento Scienze } \\
\text { Mediche e Chirurgiche e } \\
\text { Tecnologie Avanzate, GF } \\
\text { Ingrassia, Università di } \\
\text { Catania; Centro Sclerosi } \\
\text { Multipla Policlinico "G } \\
\text { Rodolico"- San Marco, } \\
\text { Università di Catania }\end{array}$ & $\begin{array}{l}\text { Site } \\
\text { Investigator }\end{array}$ & $\begin{array}{l}\text { Data } \\
\text { collection }\end{array}$ \\
\hline Viola Baione & $\begin{array}{l}\text { Department of Human } \\
\text { Neurosciences, Sapienza, } \\
\text { University of Rome }\end{array}$ & $\begin{array}{l}\text { Site } \\
\text { Investigator }\end{array}$ & $\begin{array}{l}\text { Data } \\
\text { collection }\end{array}$ \\
\hline $\begin{array}{l}\text { Roberto } \\
\text { Balgera }\end{array}$ & MS Center, ASST Lecco & $\begin{array}{l}\text { Site } \\
\text { Investigator }\end{array}$ & $\begin{array}{l}\text { Data } \\
\text { collection }\end{array}$ \\
\hline $\begin{array}{l}\text { Valeria } \\
\text { Barcella }\end{array}$ & $\begin{array}{l}\text { USS Neuroimmunologia, } \\
\text { ASST Papa Giovanni XXIII }\end{array}$ & $\begin{array}{l}\text { Site } \\
\text { Investigator }\end{array}$ & $\begin{array}{l}\text { Data } \\
\text { collection }\end{array}$ \\
\hline $\begin{array}{l}\text { Damiano } \\
\text { Baroncini }\end{array}$ & $\begin{array}{l}\text { Centro Sclerosi Multipla } \\
\text { Ospedale di Gallarate, } \\
\text { ASST della Valle Olona }\end{array}$ & $\begin{array}{l}\text { Site } \\
\text { Investigator }\end{array}$ & $\begin{array}{l}\text { Data } \\
\text { collection }\end{array}$ \\
\hline $\begin{array}{l}\text { Caterina } \\
\text { Barrilà }\end{array}$ & ASST Rhodense & $\begin{array}{l}\text { Site } \\
\text { Investigator }\end{array}$ & $\begin{array}{l}\text { Data } \\
\text { collection }\end{array}$ \\
\hline $\begin{array}{l}\text { Alessandra } \\
\text { Bellacosa }\end{array}$ & $\begin{array}{l}\text { Centro Sclerosi Multipla, UO } \\
\text { Neurologia, Ospedale San } \\
\text { Giacomo, Monopoli (Bari) }\end{array}$ & $\begin{array}{l}\text { Site } \\
\text { Investigator }\end{array}$ & $\begin{array}{l}\text { Data } \\
\text { collection }\end{array}$ \\
\hline $\begin{array}{l}\text { Gianmarco } \\
\text { Bellucci }\end{array}$ & $\begin{array}{l}\text { Department of } \\
\text { Neuroscience, Mental } \\
\text { Health and Sensory } \\
\text { Organs Sapienza } \\
\text { University S. Andrea } \\
\text { Hospital-site Rome }\end{array}$ & $\begin{array}{l}\text { Site } \\
\text { Investigator }\end{array}$ & $\begin{array}{l}\text { Data } \\
\text { collection }\end{array}$ \\
\hline $\begin{array}{l}\text { Roberto } \\
\text { Bergamaschi }\end{array}$ & $\begin{array}{l}\text { IRCCS Mondino } \\
\text { Foundation, Pavia }\end{array}$ & $\begin{array}{l}\text { Site } \\
\text { Investigator }\end{array}$ & $\begin{array}{l}\text { Data } \\
\text { collection }\end{array}$ \\
\hline $\begin{array}{l}\text { Valeria } \\
\text { Bergamaschi }\end{array}$ & $\begin{array}{l}\text { AISM Rehabilitation } \\
\text { Service Liguria }\end{array}$ & $\begin{array}{l}\text { Site } \\
\text { Investigator }\end{array}$ & $\begin{array}{l}\text { Data } \\
\text { collection }\end{array}$ \\
\hline Daiana Bezzini & $\begin{array}{l}\text { Department of Life } \\
\text { Sciences, University of } \\
\text { Siena, Siena, Italy }\end{array}$ & $\begin{array}{l}\text { Site } \\
\text { Investigator }\end{array}$ & $\begin{array}{l}\text { Data } \\
\text { collection }\end{array}$ \\
\hline Beatrice Biolzi & Centro SM Fidenza (PR) & $\begin{array}{l}\text { Site } \\
\text { Investigator }\end{array}$ & $\begin{array}{l}\text { Data } \\
\text { collection }\end{array}$ \\
\hline Alvino Bisecco & $\begin{array}{l}\text { Centro SM, I Clinica } \\
\text { Neurologica, AOU- } \\
\text { Policlinico, Università } \\
\text { della Campania "Luigi } \\
\text { Vanvitelli" }\end{array}$ & $\begin{array}{l}\text { Site } \\
\text { Investigator }\end{array}$ & $\begin{array}{l}\text { Data } \\
\text { collection }\end{array}$ \\
\hline
\end{tabular}


Appendix 2 (continued)

\begin{tabular}{|c|c|c|c|}
\hline Name & Location & Role & Contribution \\
\hline $\begin{array}{l}\text { Simona } \\
\text { Bonavita }\end{array}$ & $\begin{array}{l}\text { Department of Advanced } \\
\text { Medical and Surgical } \\
\text { Sciences, University of } \\
\text { Campania Luigi Vanvitelli, } \\
80138 \text { Naples, Italy; }\end{array}$ & $\begin{array}{l}\text { Site } \\
\text { Investigator }\end{array}$ & $\begin{array}{l}\text { Data } \\
\text { collection }\end{array}$ \\
\hline $\begin{array}{l}\text { Giovanna } \\
\text { Borriello }\end{array}$ & $\begin{array}{l}\text { NCL Isituto di } \\
\text { Neuroscienze Roma }\end{array}$ & $\begin{array}{l}\text { Site } \\
\text { Investigator }\end{array}$ & $\begin{array}{l}\text { Data } \\
\text { collection }\end{array}$ \\
\hline Chiara Bosa & $\begin{array}{l}\text { MS Center, Department } \\
\text { of Neuroscience, City of } \\
\text { Health and Science } \\
\text { University Hospital of } \\
\text { Turin, Turin, Italy }\end{array}$ & $\begin{array}{l}\text { Site } \\
\text { Investigator }\end{array}$ & $\begin{array}{l}\text { Data } \\
\text { collection }\end{array}$ \\
\hline Antonio Bosco & $\begin{array}{l}\text { Neurology Unit, } \\
\text { Department of Medical, } \\
\text { Surgical, and Health } \\
\text { Sciences, Cattinara } \\
\text { University Hospital, } \\
\text { ASUGI, Trieste }\end{array}$ & $\begin{array}{l}\text { Site } \\
\text { Investigator }\end{array}$ & $\begin{array}{l}\text { Data } \\
\text { collection }\end{array}$ \\
\hline $\begin{array}{l}\text { Francesca } \\
\text { Bovis }\end{array}$ & $\begin{array}{l}\text { Department of Health } \\
\text { Sciences, University of } \\
\text { Genoa, Genoa, Italy. }\end{array}$ & Biostatistician & $\begin{array}{l}\text { Support in } \\
\text { statistical } \\
\text { analysis }\end{array}$ \\
\hline Marco Bozzali & $\begin{array}{l}\text { Neurology II, Dept of } \\
\text { Neuroscience, University } \\
\text { of Turin }\end{array}$ & $\begin{array}{l}\text { Site } \\
\text { Investigator }\end{array}$ & $\begin{array}{l}\text { Data } \\
\text { collection }\end{array}$ \\
\hline $\begin{array}{l}\text { Laura } \\
\text { Brambilla }\end{array}$ & $\begin{array}{l}\text { Centro Sclerosi Multipla, } \\
\text { U.O Neurologia IV, } \\
\text { Fondazione IRCCS } \\
\text { Istituto Neurologico } \\
\text { "Carlo Besta", Milano }\end{array}$ & $\begin{array}{l}\text { Site } \\
\text { Investigator }\end{array}$ & $\begin{array}{l}\text { Data } \\
\text { collection }\end{array}$ \\
\hline $\begin{array}{l}\text { Maria } \\
\text { Buccafusca }\end{array}$ & $\begin{array}{l}\text { Centro Sclerosi Multipla } \\
\text { UOC Neurologia e Malattie } \\
\text { Neuromuscolari, AOU } \\
\text { Policlinico G. Martino } \\
\text { Messina }\end{array}$ & $\begin{array}{l}\text { Site } \\
\text { Investigator }\end{array}$ & $\begin{array}{l}\text { Data } \\
\text { collection }\end{array}$ \\
\hline $\begin{array}{l}\text { Elisabetta } \\
\text { Bucciantini }\end{array}$ & $\begin{array}{l}\text { Ospedale Savigliano } \\
\text { ASLCN1 }\end{array}$ & $\begin{array}{l}\text { Site } \\
\text { Investigator }\end{array}$ & $\begin{array}{l}\text { Data } \\
\text { collection }\end{array}$ \\
\hline $\begin{array}{l}\text { Sebastiano } \\
\text { Bucello }\end{array}$ & $\begin{array}{l}\text { Centro SM Ospedale } \\
\text { Muscatello Augusta } \\
\text { (ASP8 SR) }\end{array}$ & $\begin{array}{l}\text { Site } \\
\text { Investigator }\end{array}$ & $\begin{array}{l}\text { Data } \\
\text { collection }\end{array}$ \\
\hline $\begin{array}{l}\text { Maria Chiara } \\
\text { Buscarinu }\end{array}$ & $\begin{array}{l}\text { Department of } \\
\text { Neuroscience, Mental } \\
\text { Health and Sensory } \\
\text { Organs Sapienza } \\
\text { University S. Andrea } \\
\text { Hospital-site Rome }\end{array}$ & $\begin{array}{l}\text { Site } \\
\text { Investigator }\end{array}$ & $\begin{array}{l}\text { Data } \\
\text { collection }\end{array}$ \\
\hline $\begin{array}{l}\text { Maria Paola } \\
\text { Cabboi }\end{array}$ & $\begin{array}{l}\text { UOC Neurologia- centro } \\
\text { SM- AUSL- IRCCS RE }\end{array}$ & $\begin{array}{l}\text { Site } \\
\text { Investigator }\end{array}$ & $\begin{array}{l}\text { Data } \\
\text { collection }\end{array}$ \\
\hline $\begin{array}{l}\text { Massimiliano } \\
\text { Calabrese }\end{array}$ & $\begin{array}{l}\text { The Multiple Sclerosis } \\
\text { Center of University } \\
\text { Hospital of Verona Dept. } \\
\text { of Neuroscience, } \\
\text { Biomedicine and } \\
\text { Movements }\end{array}$ & $\begin{array}{l}\text { Site } \\
\text { Investigator }\end{array}$ & $\begin{array}{l}\text { Data } \\
\text { collection }\end{array}$ \\
\hline $\begin{array}{l}\text { Francesca } \\
\text { Calabria }\end{array}$ & $\begin{array}{l}\text { UO Neurologia A-Azienda } \\
\text { Ospedaliera Universitaria } \\
\text { Integrata Verona }\end{array}$ & $\begin{array}{l}\text { Site } \\
\text { Investigator }\end{array}$ & $\begin{array}{l}\text { Data } \\
\text { collection }\end{array}$ \\
\hline $\begin{array}{l}\text { Francesca } \\
\text { Caleri }\end{array}$ & $\begin{array}{l}\text { Multiple Sclerosis Center, } \\
\text { Department of } \\
\text { Neurology - Franz } \\
\text { Tappeiner Hospital } \\
\text { Meran (BZ), Italy }\end{array}$ & $\begin{array}{l}\text { Site } \\
\text { Investigator }\end{array}$ & $\begin{array}{l}\text { Data } \\
\text { collection }\end{array}$ \\
\hline
\end{tabular}

Appendix 2 (continued)

\begin{tabular}{llll}
\hline Name & Location & Role & Contribution \\
\hline Federico & IRCCS Istituto delle & Site & Data \\
Camilli & Scienze Neurologiche di & Investigator & collection \\
& Bologna, UOSI & & \\
& Riabilitazione Sclerosi & & \\
& Multipla & & \\
& Cen & &
\end{tabular}

\begin{tabular}{|c|c|c|c|}
\hline $\begin{array}{l}\text { Luisa Maria } \\
\text { Caniatti }\end{array}$ & $\begin{array}{l}\text { Centro sclerosi Multipla } \\
\text { azienda ospedaliera } \\
\text { universitaria S. Anna, } \\
\text { Ferrara }\end{array}$ & $\begin{array}{l}\text { Site } \\
\text { Investigator }\end{array}$ & $\begin{array}{l}\text { Data } \\
\text { collection }\end{array}$ \\
\hline $\begin{array}{l}\text { Roberto } \\
\text { Cantello }\end{array}$ & $\begin{array}{l}\text { Neurology Unit, } \\
\text { Maggiore della Carità } \\
\text { Hospital, Department of } \\
\text { Translational Medicine, } \\
\text { University of Piemonte } \\
\text { Orientale, Novara, Italy }\end{array}$ & $\begin{array}{l}\text { Site } \\
\text { Investigator }\end{array}$ & $\begin{array}{l}\text { Data } \\
\text { collection }\end{array}$ \\
\hline Ruggero Capra & $\begin{array}{l}\text { Centro Sclerosi Multipla } \\
\text { ASST Spedali Civili di } \\
\text { Brescia, Ospedale di } \\
\text { Montichiari }\end{array}$ & $\begin{array}{l}\text { Site } \\
\text { Investigator }\end{array}$ & $\begin{array}{l}\text { Data } \\
\text { collection }\end{array}$ \\
\hline $\begin{array}{l}\text { Rocco } \\
\text { Capuano }\end{array}$ & $\begin{array}{l}\text { Centro SM, I Clinica } \\
\text { Neurologica, AOU- } \\
\text { Policlinico, Università } \\
\text { della Campania "Luigi } \\
\text { Vanvitelli" }\end{array}$ & $\begin{array}{l}\text { Site } \\
\text { Investigator }\end{array}$ & $\begin{array}{l}\text { Data } \\
\text { collection }\end{array}$ \\
\hline
\end{tabular}

\begin{tabular}{|c|c|c|c|}
\hline Patrizia Carta & $\begin{array}{l}\text { Centro Sclerosi Multipla } \\
\text { Ospedale di Gallarate, } \\
\text { ASST della Valle Olona }\end{array}$ & $\begin{array}{l}\text { Site } \\
\text { Investigator }\end{array}$ & $\begin{array}{l}\text { Data } \\
\text { collection }\end{array}$ \\
\hline $\begin{array}{l}\text { Maria Grazia } \\
\text { Celani }\end{array}$ & $\begin{array}{l}\text { Azienda Ospedaliera di } \\
\text { Perugia, SC di } \\
\text { Neurofisiopatologia }\end{array}$ & $\begin{array}{l}\text { Site } \\
\text { Investigator }\end{array}$ & $\begin{array}{l}\text { Data } \\
\text { collection }\end{array}$ \\
\hline $\begin{array}{l}\text { Maria } \\
\text { Cellerino }\end{array}$ & $\begin{array}{l}\text { DINOGMI Universita' di } \\
\text { Genova }\end{array}$ & $\begin{array}{l}\text { Site } \\
\text { Investigator }\end{array}$ & $\begin{array}{l}\text { Data } \\
\text { collection }\end{array}$ \\
\hline $\begin{array}{l}\text { Raffaella } \\
\text { Cerqua }\end{array}$ & $\begin{array}{l}\text { Clinica Neurologica } \\
\text { Ospedali Riuniti Ancona }\end{array}$ & $\begin{array}{l}\text { Site } \\
\text { Investigator }\end{array}$ & $\begin{array}{l}\text { Data } \\
\text { collection }\end{array}$ \\
\hline Clara Chisari & $\begin{array}{l}\text { Dipartimento Scienze } \\
\text { Mediche e Chirurgiche } \\
\text { e Tecnologie Avanzate, } \\
\text { GF Ingrassia, Università } \\
\text { di Catania; Centro } \\
\text { Sclerosi Multipla } \\
\text { Policlinico "G Rodolico"- } \\
\text { San Marco, Università } \\
\text { di Catania }\end{array}$ & $\begin{array}{l}\text { Site } \\
\text { Investigator }\end{array}$ & $\begin{array}{l}\text { Data } \\
\text { collection }\end{array}$ \\
\hline
\end{tabular}

\begin{tabular}{llll}
\hline $\begin{array}{l}\text { Raffaella } \\
\text { Clerici }\end{array}$ & $\begin{array}{l}\text { Centro Sclerosi Multipla } \\
\text { U.O. Neurologia } \\
\text { Ospedale Valduce Como }\end{array}$ & $\begin{array}{l}\text { Site } \\
\text { Investigator }\end{array}$ & $\begin{array}{l}\text { Data } \\
\text { collection }\end{array}$ \\
\hline $\begin{array}{l}\text { Marinella } \\
\text { Clerico }\end{array}$ & $\begin{array}{l}\text { Clinical and Biological } \\
\text { Sciences Dept, University } \\
\text { of Torino }\end{array}$ & $\begin{array}{l}\text { Site } \\
\text { Investigator }\end{array}$ & $\begin{array}{l}\text { Data } \\
\text { collection }\end{array}$ \\
\hline Gaia Cola & $\begin{array}{l}\text { Multiple Sclerosis Clinical } \\
\text { and Research Unit, } \\
\text { Department of Systems } \\
\text { Medicine, Tor Vergata } \\
\text { University, Rome, Italy }\end{array}$ & $\begin{array}{l}\text { Site } \\
\text { Investigator }\end{array}$ & $\begin{array}{l}\text { Data } \\
\text { collection }\end{array}$ \\
\hline $\begin{array}{l}\text { Antonella } \\
\text { Conte }\end{array}$ & $\begin{array}{l}\text { 1) Department of Human } \\
\text { Neurosciences, Sapienza, }\end{array}$ & $\begin{array}{l}\text { Site } \\
\text { Investigator }\end{array}$ & $\begin{array}{l}\text { Data } \\
\text { collection }\end{array}$ \\
& $\begin{array}{l}\text { University of Rome. 2) } \\
\text { IRCCS Neuromed, Pozzilli } \\
\text { (IS) }\end{array}$ & & \\
\hline $\begin{array}{l}\text { Marta Zaffira } \\
\text { Conti }\end{array}$ & $\begin{array}{l}\text { USS Neuroimmunologia, } \\
\text { ASST Papa Giovanni XXIII }\end{array}$ & $\begin{array}{l}\text { Site } \\
\text { Investigator }\end{array}$ & $\begin{array}{l}\text { Data } \\
\text { collection }\end{array}$ \\
\hline
\end{tabular}


Appendix 2 (continued)

\begin{tabular}{|c|c|c|c|c|c|c|c|}
\hline Name & Location & Role & Contribution & Name & Location & Role & Contribution \\
\hline $\begin{array}{l}\text { Christian } \\
\text { Cordano }\end{array}$ & UCSF, San Francisco, USA & $\begin{array}{l}\text { Site } \\
\text { Investigator }\end{array}$ & $\begin{array}{l}\text { Data } \\
\text { collection }\end{array}$ & \multirow[t]{2}{*}{$\begin{array}{l}\text { Nicola De } \\
\text { Stefano }\end{array}$} & \multirow{2}{*}{$\begin{array}{l}\text { Department of Medicine, } \\
\text { Surgery and } \\
\text { Neuroscience, University } \\
\text { of Siena, Italy }\end{array}$} & \multirow[t]{2}{*}{$\begin{array}{l}\text { Site } \\
\text { Investigator }\end{array}$} & \multirow[t]{2}{*}{$\begin{array}{l}\text { Data } \\
\text { collection }\end{array}$} \\
\hline Susanna & SC Neurologia Ausl Valle & & Data & & & & \\
\hline $\begin{array}{l}\text { Francesco } \\
\text { Corea }\end{array}$ & $\begin{array}{l}\text { Neurologia, Ospedale } \\
\text { San Giovanni, Foligno }\end{array}$ & $\begin{array}{l}\text { Site } \\
\text { Investigator }\end{array}$ & $\begin{array}{l}\text { Data } \\
\text { collection }\end{array}$ & $\begin{array}{l}\text { Marco Della } \\
\text { Cava }\end{array}$ & $\begin{array}{l}\text { AISM Padova } \\
\text { Rehabilitation Service }\end{array}$ & $\begin{array}{l}\text { Site } \\
\text { Investigator }\end{array}$ & $\begin{array}{l}\text { Data } \\
\text { collection }\end{array}$ \\
\hline Claudio & AISM Vicenza & Site & Data & $\begin{array}{l}\text { Mario di } \\
\text { Napoli }\end{array}$ & $\begin{array}{l}\text { Centro Sclerosi Multipla } \\
\text { Rieti (Rieti) }\end{array}$ & $\begin{array}{l}\text { Site } \\
\text { Investigator }\end{array}$ & $\begin{array}{l}\text { Data } \\
\text { collection }\end{array}$ \\
\hline Correale & Rehabilitation Service & Investigator & collection & \multirow{2}{*}{$\begin{array}{l}\text { Alessia Di } \\
\text { Sapio }\end{array}$} & \multirow[b]{2}{*}{$\begin{array}{l}\text { Department of } \\
\text { Neurology, Regina } \\
\text { Montis Regalis Hospital, } \\
\text { Mondovi (CN) }\end{array}$} & \multirow{2}{*}{$\begin{array}{l}\text { Site } \\
\text { Investigator }\end{array}$} & \multirow{2}{*}{$\begin{array}{l}\text { Data } \\
\text { collection }\end{array}$} \\
\hline $\begin{array}{l}\text { Salvatore } \\
\text { Cottone }\end{array}$ & $\begin{array}{l}\text { Centro Sclerosi Multipla } \\
\text { U.O.C. Neurologia con } \\
\text { Stroke Unit A.R.N.A.S } \\
\text { Civico (Palermo) }\end{array}$ & $\begin{array}{l}\text { Site } \\
\text { Investigator }\end{array}$ & $\begin{array}{l}\text { Data } \\
\text { collection }\end{array}$ & & & & \\
\hline \multirow[t]{2}{*}{$\begin{array}{l}\text { Francesco } \\
\text { Crescenzo }\end{array}$} & \multirow{2}{*}{$\begin{array}{l}\text { The Multiple Sclerosis } \\
\text { Center of University } \\
\text { Hospital of Verona Dept. } \\
\text { of Neuroscience, } \\
\text { Biomedicine and } \\
\text { Movements }\end{array}$} & $\begin{array}{l}\text { Site } \\
\text { Investigator }\end{array}$ & $\begin{array}{l}\text { Data } \\
\text { collection }\end{array}$ & $\begin{array}{l}\text { Renato } \\
\text { Docimo }\end{array}$ & $\begin{array}{l}\text { Centro Sclerosi Multipla, } \\
\text { Presidio Ospedaliero "San } \\
\text { Giuseppe Moscati" ASL } \\
\text { Caserta, Aversa (CE). }\end{array}$ & $\begin{array}{l}\text { Site } \\
\text { Investigator }\end{array}$ & $\begin{array}{l}\text { Data } \\
\text { collection }\end{array}$ \\
\hline & & & & Anna Dutto & $\begin{array}{l}\text { Ospedale Savigliano } \\
\text { Aslcn1 }\end{array}$ & $\begin{array}{l}\text { Site } \\
\text { Investigator }\end{array}$ & $\begin{array}{l}\text { Data } \\
\text { collection }\end{array}$ \\
\hline \multirow[t]{2}{*}{ Erica Curti } & \multirow{2}{*}{$\begin{array}{l}\text { Multiple Sclerosis Centre, } \\
\text { Department of General } \\
\text { Medicine, Parma } \\
\text { University Hospital, } \\
\text { Parma }\end{array}$} & \multirow[t]{2}{*}{$\begin{array}{l}\text { Site } \\
\text { Investigator }\end{array}$} & \multirow[t]{2}{*}{$\begin{array}{l}\text { Data } \\
\text { collection }\end{array}$} & $\begin{array}{l}\text { Luana } \\
\text { Evangelista }\end{array}$ & $\begin{array}{l}\text { Demyelinating Disease } \\
\text { Center, San Salvatore } \\
\text { Hospital, L'Aquila }\end{array}$ & $\begin{array}{l}\text { Site } \\
\text { Investigator }\end{array}$ & $\begin{array}{l}\text { Data } \\
\text { collection }\end{array}$ \\
\hline & & & & \multirow[t]{2}{*}{$\begin{array}{l}\text { Salvatore } \\
\text { Fanara }\end{array}$} & \multirow{2}{*}{$\begin{array}{l}\text { Department of } \\
\text { Biomedicine, } \\
\text { Neurosciences and } \\
\text { Advanced Diagnsotics; } \\
\text { University of Palermo }\end{array}$} & \multirow[t]{2}{*}{$\begin{array}{l}\text { Site } \\
\text { Investigator }\end{array}$} & \multirow[t]{2}{*}{$\begin{array}{l}\text { Data } \\
\text { collection }\end{array}$} \\
\hline \multirow[t]{2}{*}{$\begin{array}{l}\text { Alessandro } \\
\text { d'Ambrosio }\end{array}$} & \multirow{2}{*}{$\begin{array}{l}\text { Centro SM, I Clinica } \\
\text { Neurologica, AOU- } \\
\text { Policlinico, Università } \\
\text { della Campania "Luigi } \\
\text { Vanvitelli" }\end{array}$} & \multirow[t]{2}{*}{$\begin{array}{l}\text { Site } \\
\text { Investigator }\end{array}$} & \multirow[t]{2}{*}{$\begin{array}{l}\text { Data } \\
\text { collection }\end{array}$} & & & & \\
\hline & & & & Diana Ferraro & $\begin{array}{l}\text { Department of } \\
\text { Biomedical, Metabolic }\end{array}$ & $\begin{array}{l}\text { Site } \\
\text { Investigator }\end{array}$ & $\begin{array}{l}\text { Data } \\
\text { collection }\end{array}$ \\
\hline $\begin{array}{l}\text { Emanuele } \\
\text { D'Amico }\end{array}$ & $\begin{array}{l}\text { Dipartimento Scienze } \\
\text { Mediche e Chirurgiche e } \\
\text { Tecnologie Avanzate, GF } \\
\text { Ingrassia, Università di } \\
\text { Catania; Centro Sclerosi }\end{array}$ & $\begin{array}{l}\text { Site } \\
\text { Investigator }\end{array}$ & $\begin{array}{l}\text { Data } \\
\text { collection }\end{array}$ & & $\begin{array}{l}\text { and Neurosciences, } \\
\text { University of Modena } \\
\text { and Reggio Emilia, } \\
\text { Modena, Italy }\end{array}$ & & \\
\hline & $\begin{array}{l}\text { Multipla Policlinico "G } \\
\text { Rodolico"- San Marco, } \\
\text { Università di Catania }\end{array}$ & & & $\begin{array}{l}\text { Maria Teresa } \\
\text { Ferrò }\end{array}$ & $\begin{array}{l}\text { Neuroimmunology, } \\
\text { Center for Multiple } \\
\text { Sclerosis, ASST, Crema, } \\
\text { Italy }\end{array}$ & $\begin{array}{l}\text { Site } \\
\text { Investigator }\end{array}$ & $\begin{array}{l}\text { Data } \\
\text { collection }\end{array}$ \\
\hline $\begin{array}{l}\text { Maura Chiara } \\
\text { Danni }\end{array}$ & $\begin{array}{l}\text { Clinica Neurologica } \\
\text { Ospedali Riuniti Ancona }\end{array}$ & $\begin{array}{l}\text { Site } \\
\text { Investigator }\end{array}$ & $\begin{array}{l}\text { Data } \\
\text { collection }\end{array}$ & $\begin{array}{l}\text { Cristina } \\
\text { Fioretti }\end{array}$ & UO Neurologia Livorno & $\begin{array}{l}\text { Site } \\
\text { Investigator }\end{array}$ & $\begin{array}{l}\text { Data } \\
\text { collection }\end{array}$ \\
\hline Alessia d'Arma & $\begin{array}{l}\text { IRCCS Fondazione Don } \\
\text { Carlo Gnocchi ONLUS, } \\
\text { Milano }\end{array}$ & $\begin{array}{l}\text { Site } \\
\text { Investigator }\end{array}$ & $\begin{array}{l}\text { Data } \\
\text { collection }\end{array}$ & Mario Fratta & $\begin{array}{l}\text { Il Clinica Neurologica, } \\
\text { Università della }\end{array}$ & $\begin{array}{l}\text { Site } \\
\text { Investigator }\end{array}$ & $\begin{array}{l}\text { Data } \\
\text { collection }\end{array}$ \\
\hline $\begin{array}{l}\text { Vincenzo } \\
\text { Dattola }\end{array}$ & $\begin{array}{l}\text { UOC Neurologia, Grande } \\
\text { Ospedale Metropolitano }\end{array}$ & $\begin{array}{l}\text { Site } \\
\text { Investigator }\end{array}$ & $\begin{array}{l}\text { Data } \\
\text { collection }\end{array}$ & & Vanvitelli, Naples & & \\
\hline & $\begin{array}{l}\text { "Bianchi Melacrino } \\
\text { Morelli", Reggio di } \\
\text { Calabria }\end{array}$ & & & Jessica Frau & $\begin{array}{l}\text { Centro Sclerosi Multipla, } \\
\text { ATS Sardegna/ Dpt } \\
\text { Scienze Mediche e } \\
\text { Sanità Publica. }\end{array}$ & $\begin{array}{l}\text { Site } \\
\text { Investigator }\end{array}$ & $\begin{array}{l}\text { Data } \\
\text { collection }\end{array}$ \\
\hline $\begin{array}{l}\text { Stefano de } \\
\text { Biase }\end{array}$ & $\begin{array}{l}\text { Neurology Unit, } \\
\text { Ospedale dell'Angelo, } \\
\text { Venezia-Mestre, Italy }\end{array}$ & $\begin{array}{l}\text { Site } \\
\text { Investigator }\end{array}$ & $\begin{array}{l}\text { Data } \\
\text { collection }\end{array}$ & & $\begin{array}{l}\text { Università di Cagliari, } \\
\text { Cagliari }\end{array}$ & & \\
\hline $\begin{array}{l}\text { Giovanna De } \\
\text { Luca }\end{array}$ & $\begin{array}{l}\text { MS Centre, Department } \\
\text { of Clinical Neurology, SS. } \\
\text { Annunziata, University } \\
\text { Hospital, Chieti, Italy }\end{array}$ & $\begin{array}{l}\text { Site } \\
\text { Investigator }\end{array}$ & $\begin{array}{l}\text { Data } \\
\text { collection }\end{array}$ & Marzia Fronza & $\begin{array}{l}\text { Centro Sclerosi Multipla, } \\
\text { ATS Sardegna/ Dpt } \\
\text { Scienze Mediche e Sanità } \\
\text { Publica, Università di } \\
\text { Cagliari, Cagliari }\end{array}$ & $\begin{array}{l}\text { Site } \\
\text { Investigator }\end{array}$ & $\begin{array}{l}\text { Data } \\
\text { collection }\end{array}$ \\
\hline $\begin{array}{l}\text { Stefania } \\
\text { Federica De } \\
\text { Mercanti }\end{array}$ & $\begin{array}{l}\text { Clinical and Biological } \\
\text { Sciences Dept, University } \\
\text { of Torino }\end{array}$ & $\begin{array}{l}\text { Site } \\
\text { Investigator }\end{array}$ & $\begin{array}{l}\text { Data } \\
\text { collection }\end{array}$ & Roberto Furlan & $\begin{array}{l}\text { Institute of Experimental } \\
\text { Neurology, Division of } \\
\text { Neuroscience, IRCCS } \\
\text { Ospedale San Raffaele, }\end{array}$ & $\begin{array}{l}\text { Site } \\
\text { Investigator }\end{array}$ & $\begin{array}{l}\text { Data } \\
\text { collection }\end{array}$ \\
\hline Paolo De Mitri & $\begin{array}{l}\text { Emergency Department, } \\
\text { Guglielmo da Saliceto } \\
\text { Hospital, Piacenza, Italy }\end{array}$ & $\begin{array}{l}\text { Site } \\
\text { Investigator }\end{array}$ & $\begin{array}{l}\text { Data } \\
\text { collection }\end{array}$ & & $\begin{array}{l}\text { Milano, Italy, and Italian } \\
\text { Neuroimmunology } \\
\text { Association-AINI }\end{array}$ & & \\
\hline
\end{tabular}

Appendix 2 (continued) 
Appendix 2 (continued)

\begin{tabular}{|c|c|c|c|}
\hline Name & Location & Role & Contribution \\
\hline $\begin{array}{l}\text { Alberto } \\
\text { Gajofatto }\end{array}$ & $\begin{array}{l}\text { Dipartimento di } \\
\text { Neuroscienze, } \\
\text { Biomedicina e } \\
\text { Movimento, Università di } \\
\text { Verona }\end{array}$ & $\begin{array}{l}\text { Site } \\
\text { Investigator }\end{array}$ & $\begin{array}{l}\text { Data } \\
\text { collection }\end{array}$ \\
\hline Antonio Gallo & $\begin{array}{l}\text { Centro SM, I Clinica } \\
\text { Neurologica, AOU- } \\
\text { Policlinico, Università } \\
\text { della Campania "Luigi } \\
\text { Vanvitelli" }\end{array}$ & $\begin{array}{l}\text { Site } \\
\text { Investigator }\end{array}$ & $\begin{array}{l}\text { Data } \\
\text { collection }\end{array}$ \\
\hline Paolo Gallo & $\begin{array}{l}\text { Multiple Sclerosis Centre of } \\
\text { the Veneto Region } \\
\text { (CeSMuV), University } \\
\text { Hospital of Padua, Italy. }\end{array}$ & $\begin{array}{l}\text { Site } \\
\text { Investigator }\end{array}$ & $\begin{array}{l}\text { Data } \\
\text { collection }\end{array}$ \\
\hline $\begin{array}{l}\text { Claudio } \\
\text { Gasperini }\end{array}$ & $\begin{array}{l}\text { Dept Neurosciences, San } \\
\text { Camillo Forlanini } \\
\text { Hospital - Rome }\end{array}$ & $\begin{array}{l}\text { Site } \\
\text { Investigator }\end{array}$ & $\begin{array}{l}\text { Data } \\
\text { collection }\end{array}$ \\
\hline $\begin{array}{l}\text { Anna } \\
\text { Ghazaryan }\end{array}$ & UO Neurologia & $\begin{array}{l}\text { Site } \\
\text { Investigator }\end{array}$ & $\begin{array}{l}\text { Data } \\
\text { collection }\end{array}$ \\
\hline $\begin{array}{l}\text { Bruno } \\
\text { Giometto }\end{array}$ & $\begin{array}{l}\text { Ospedale Santa Chiara, } \\
\text { Trento. UO Neurologia } \\
\text { (Trento) }\end{array}$ & $\begin{array}{l}\text { Site } \\
\text { Investigator }\end{array}$ & $\begin{array}{l}\text { Data } \\
\text { collection }\end{array}$ \\
\hline $\begin{array}{l}\text { Francesca } \\
\text { Gobbin }\end{array}$ & $\begin{array}{l}\text { Dipartimento di } \\
\text { Neuroscienze, } \\
\text { Biomedicina e } \\
\text { Movimento, Università di } \\
\text { Verona }\end{array}$ & $\begin{array}{l}\text { Site } \\
\text { Investigator }\end{array}$ & $\begin{array}{l}\text { Data } \\
\text { collection }\end{array}$ \\
\hline Flora Govone & $\begin{array}{l}\text { Department of Neurology, } \\
\text { Regina Montis Regalis } \\
\text { Hospital, Mondovi (CN) }\end{array}$ & $\begin{array}{l}\text { Site } \\
\text { Investigator }\end{array}$ & $\begin{array}{l}\text { Data } \\
\text { collection }\end{array}$ \\
\hline $\begin{array}{l}\text { Franco } \\
\text { Granella }\end{array}$ & $\begin{array}{l}\text { Unit of Neurosciences, } \\
\text { Department of Medicine } \\
\text { and Surgery, University of } \\
\text { Parma, Parma \& Multiple } \\
\text { Sclerosis Centre, } \\
\text { Department of General } \\
\text { Medicine, Parma University } \\
\text { Hospital, Parma }\end{array}$ & $\begin{array}{l}\text { Site } \\
\text { Investigator }\end{array}$ & $\begin{array}{l}\text { Data } \\
\text { collection }\end{array}$ \\
\hline Erica Grange & $\begin{array}{l}\text { Dept. of Rehabilitation, } \\
\text { CRRF "Mons. Luigi } \\
\text { Novarese", Moncrivello, } \\
\text { Italy }\end{array}$ & $\begin{array}{l}\text { Site } \\
\text { Investigator }\end{array}$ & $\begin{array}{l}\text { Data } \\
\text { collection }\end{array}$ \\
\hline $\begin{array}{l}\text { Maria Grazia } \\
\text { Grasso }\end{array}$ & $\begin{array}{l}\text { IRCCS Fondazione Santa } \\
\text { Lucia }\end{array}$ & $\begin{array}{l}\text { Site } \\
\text { Investigator }\end{array}$ & $\begin{array}{l}\text { Data } \\
\text { collection }\end{array}$ \\
\hline $\begin{array}{l}\text { Angelica } \\
\text { Guareschi }\end{array}$ & Centro SM Fidenza (PR) & $\begin{array}{l}\text { Site } \\
\text { Investigator }\end{array}$ & $\begin{array}{l}\text { Data } \\
\text { collection }\end{array}$ \\
\hline $\begin{array}{l}\text { Clara } \\
\text { Guaschino }\end{array}$ & $\begin{array}{l}\text { Centro Sclerosi Multipla } \\
\text { Ospedale di Gallarate, } \\
\text { ASST della Valle Olona }\end{array}$ & $\begin{array}{l}\text { Site } \\
\text { Investigator }\end{array}$ & $\begin{array}{l}\text { Data } \\
\text { collection }\end{array}$ \\
\hline $\begin{array}{l}\text { Simone } \\
\text { Guerrieri }\end{array}$ & $\begin{array}{l}\text { Neurology Department, } \\
\text { Multiple Sclerosis Center, } \\
\text { San Raffaele Hospital, } \\
\text { Milan }\end{array}$ & $\begin{array}{l}\text { Site } \\
\text { Investigator }\end{array}$ & $\begin{array}{l}\text { Data } \\
\text { collection }\end{array}$ \\
\hline $\begin{array}{l}\text { Donata } \\
\text { Guidetti }\end{array}$ & $\begin{array}{l}\text { Emergency Department, } \\
\text { Guglielmo da Saliceto } \\
\text { Hospital, Piacenza, Italy }\end{array}$ & $\begin{array}{l}\text { Site } \\
\text { Investigator }\end{array}$ & $\begin{array}{l}\text { Data } \\
\text { collection }\end{array}$ \\
\hline $\begin{array}{l}\text { Pietro } \\
\text { laffaldano }\end{array}$ & $\begin{array}{l}\text { Department of Basic } \\
\text { Medical Sciences, } \\
\text { Neurosciences and } \\
\text { Sense Organs - University } \\
\text { of Bari Aldo Moro }\end{array}$ & $\begin{array}{l}\text { Site } \\
\text { Investigator }\end{array}$ & $\begin{array}{l}\text { Data } \\
\text { collection }\end{array}$ \\
\hline
\end{tabular}

Appendix 2 (continued)

\begin{tabular}{|c|c|c|c|}
\hline Name & Location & Role & Contribution \\
\hline $\begin{array}{l}\text { Antonio } \\
\text { Ianniello }\end{array}$ & $\begin{array}{l}\text { Centro SM S.Andrea Dip. } \\
\text { Neuroscienze Umane } \\
\text { Sapienza Roma }\end{array}$ & $\begin{array}{l}\text { Site } \\
\text { Investigator }\end{array}$ & $\begin{array}{l}\text { Data } \\
\text { collection }\end{array}$ \\
\hline Luigi lasevoli & $\begin{array}{l}\text { IRCCS Fondazione Santa } \\
\text { Lucia }\end{array}$ & $\begin{array}{l}\text { Site } \\
\text { Investigator }\end{array}$ & $\begin{array}{l}\text { Data } \\
\text { collection }\end{array}$ \\
\hline $\begin{array}{l}\text { Daniele } \\
\text { Imperiale }\end{array}$ & $\begin{array}{l}\text { SC Neurologia1 Ospedale } \\
\text { Maria Vittoria- Torino }\end{array}$ & $\begin{array}{l}\text { Site } \\
\text { Investigator }\end{array}$ & $\begin{array}{l}\text { Data } \\
\text { collection }\end{array}$ \\
\hline $\begin{array}{l}\text { Maria Teresa } \\
\text { Infante }\end{array}$ & $\begin{array}{l}\text { Neurologia ASL } 1 \\
\text { imperiese }\end{array}$ & $\begin{array}{l}\text { Site } \\
\text { Investigator }\end{array}$ & $\begin{array}{l}\text { Data } \\
\text { collection }\end{array}$ \\
\hline Rosa lodice & $\begin{array}{l}\text { Department of } \\
\text { Neuroscience, } \\
\text { Reproductive Sciences } \\
\text { and Odontostomatology, } \\
\text { University Federico II of } \\
\text { Naples, Naples, Italy }\end{array}$ & $\begin{array}{l}\text { Site } \\
\text { Investigator }\end{array}$ & $\begin{array}{l}\text { Data } \\
\text { collection }\end{array}$ \\
\hline Aniello lovino & $\begin{array}{l}\text { Department of Advanced } \\
\text { Biomedical Sciences, } \\
\text { University Federico II, } \\
\text { Naples, Italy }\end{array}$ & $\begin{array}{l}\text { Site } \\
\text { Investigator }\end{array}$ & $\begin{array}{l}\text { Data } \\
\text { collection }\end{array}$ \\
\hline $\begin{array}{l}\text { Giovanna } \\
\text { Konrad }\end{array}$ & $\begin{array}{l}\text { AISM Aosta } \\
\text { Rehabilitation Service }\end{array}$ & $\begin{array}{l}\text { Site } \\
\text { Investigator }\end{array}$ & $\begin{array}{l}\text { Data } \\
\text { collection }\end{array}$ \\
\hline Doriana Landi & $\begin{array}{l}\text { Multiple Sclerosis Clinical } \\
\text { and Research Unit, } \\
\text { Department of Systems } \\
\text { Medicine, Tor Vergata } \\
\text { University, Rome, Italy }\end{array}$ & $\begin{array}{l}\text { Site } \\
\text { Investigator }\end{array}$ & $\begin{array}{l}\text { Data } \\
\text { collection }\end{array}$ \\
\hline $\begin{array}{l}\text { Roberta } \\
\text { Lanzillo }\end{array}$ & $\begin{array}{l}\text { Federico II University of } \\
\text { Naples }\end{array}$ & $\begin{array}{l}\text { Site } \\
\text { Investigator }\end{array}$ & $\begin{array}{l}\text { Data } \\
\text { collection }\end{array}$ \\
\hline $\begin{array}{l}\text { Caterina } \\
\text { Lapucci }\end{array}$ & $\begin{array}{l}\text { DINOGMI Universita' di } \\
\text { Genova }\end{array}$ & $\begin{array}{l}\text { Site } \\
\text { Investigator }\end{array}$ & $\begin{array}{l}\text { Data } \\
\text { collection }\end{array}$ \\
\hline Luigi Lavorgna & $\begin{array}{l}\text { Department of Advanced } \\
\text { Medical and Surgical } \\
\text { Sciences, University of } \\
\text { Campania Luigi } \\
\text { Vanvitelli, } 80138 \text { Naples, } \\
\text { Italy; }\end{array}$ & $\begin{array}{l}\text { Site } \\
\text { Investigator }\end{array}$ & $\begin{array}{l}\text { Data } \\
\text { collection }\end{array}$ \\
\hline $\begin{array}{l}\text { Maria Rita } \\
\text { L'Episcopo }\end{array}$ & $\begin{array}{l}\text { Centro Sclerosi Multipla } \\
\text { ospedale San Lazzaro } \\
\text { (Alba, CN) }\end{array}$ & $\begin{array}{l}\text { Site } \\
\text { Investigator }\end{array}$ & $\begin{array}{l}\text { Data } \\
\text { collection }\end{array}$ \\
\hline Serena Leva & $\begin{array}{l}\text { Centro Sclerosi Multipla, } \\
\text { Ospedale di Legnano, } \\
\text { ASST OVEST MI, Italia }\end{array}$ & $\begin{array}{l}\text { Site } \\
\text { Investigator }\end{array}$ & $\begin{array}{l}\text { Data } \\
\text { collection }\end{array}$ \\
\hline $\begin{array}{l}\text { Giuseppe } \\
\text { Liberatore }\end{array}$ & $\begin{array}{l}\text { Neuromuscular and } \\
\text { Neuroimmunology } \\
\text { Service, IRCCS } \\
\text { Humanitas Clinical and } \\
\text { Research Institute, } \\
\text { Rozzano, Milan, Italy }\end{array}$ & $\begin{array}{l}\text { Site } \\
\text { Investigator }\end{array}$ & $\begin{array}{l}\text { Data } \\
\text { collection }\end{array}$ \\
\hline $\begin{array}{l}\text { Marianna Lo } \\
\operatorname{Re}\end{array}$ & $\begin{array}{l}\text { SCDO Neurologia e } \\
\text { Centro di Riferimento } \\
\text { Regionale Sclerosi } \\
\text { Multipla, AOU San Luigi - } \\
\text { Orbassano (TO) }\end{array}$ & $\begin{array}{l}\text { Site } \\
\text { Investigator }\end{array}$ & $\begin{array}{l}\text { Data } \\
\text { collection }\end{array}$ \\
\hline Marco Longoni & $\begin{array}{l}\text { Local Health Agency of } \\
\text { Romagna, Maurizio } \\
\text { Bufalini Hospital } \\
\text { (Cesena) - Neurology Unit }\end{array}$ & $\begin{array}{l}\text { Site } \\
\text { Investigator }\end{array}$ & $\begin{array}{l}\text { Data } \\
\text { collection }\end{array}$ \\
\hline $\begin{array}{l}\text { Leonardo } \\
\text { Lopiano }\end{array}$ & $\begin{array}{l}\text { Neurology II, Dept of } \\
\text { Neuroscience, University } \\
\text { of Turin }\end{array}$ & $\begin{array}{l}\text { Site } \\
\text { Investigator }\end{array}$ & $\begin{array}{l}\text { Data } \\
\text { collection }\end{array}$ \\
\hline
\end{tabular}

Continued 
Appendix 2 (continued)

\begin{tabular}{|c|c|c|c|}
\hline Name & Location & Role & Contribution \\
\hline $\begin{array}{l}\text { Lorena } \\
\text { Lorefice }\end{array}$ & $\begin{array}{l}\text { Centro Sclerosi Multipla, } \\
\text { ATS Sardegna/ Dpt } \\
\text { Scienze Mediche e } \\
\text { Sanità Publica, } \\
\text { Università di Cagliari, } \\
\text { Cagliari }\end{array}$ & $\begin{array}{l}\text { Site } \\
\text { Investigator }\end{array}$ & $\begin{array}{l}\text { Data } \\
\text { collection }\end{array}$ \\
\hline $\begin{array}{l}\text { Matteo } \\
\text { Lucchini }\end{array}$ & $\begin{array}{l}\text { Fondazione Policlinico } \\
\text { Universitario Agostino } \\
\text { Gemelli IRCCS - } \\
\text { Università Cattolica del } \\
\text { Sacro Cuore }\end{array}$ & $\begin{array}{l}\text { Site } \\
\text { Investigator }\end{array}$ & $\begin{array}{l}\text { Data } \\
\text { collection }\end{array}$ \\
\hline Giacomo Lus & $\begin{array}{l}\text { Il Clinica Neurologica, } \\
\text { Università della } \\
\text { Campania Luigi } \\
\text { Vanvitelli, Naples }\end{array}$ & $\begin{array}{l}\text { Site } \\
\text { Investigator }\end{array}$ & $\begin{array}{l}\text { Data } \\
\text { collection }\end{array}$ \\
\hline $\begin{array}{l}\text { Davide } \\
\text { Maimone }\end{array}$ & $\begin{array}{l}\text { Centro SM - UOC } \\
\text { Neurologia - ARNAS } \\
\text { Garibaldi - Catania }\end{array}$ & $\begin{array}{l}\text { Site } \\
\text { Investigator }\end{array}$ & $\begin{array}{l}\text { Data } \\
\text { collection }\end{array}$ \\
\hline $\begin{array}{l}\text { Maria } \\
\text { Malentacchi }\end{array}$ & $\begin{array}{l}\text { SCDO Neurologia e } \\
\text { Centro di Riferimento } \\
\text { Regionale Sclerosi } \\
\text { Multipla, AOU San Luigi - } \\
\text { Orbassano (TO) }\end{array}$ & $\begin{array}{l}\text { Site } \\
\text { Investigator }\end{array}$ & $\begin{array}{l}\text { Data } \\
\text { collection }\end{array}$ \\
\hline Giulia Mallucci & $\begin{array}{l}\text { IRCCS Mondino } \\
\text { Foundation, Pavia }\end{array}$ & $\begin{array}{l}\text { Site } \\
\text { Investigator }\end{array}$ & $\begin{array}{l}\text { Data } \\
\text { collection }\end{array}$ \\
\hline $\begin{array}{l}\text { Simona } \\
\text { Malucchi }\end{array}$ & $\begin{array}{l}\text { SCDO Neurologia e } \\
\text { Centro di Riferimento } \\
\text { Regionale Sclerosi } \\
\text { Multipla, AOU San Luigi - } \\
\text { Orbassano (TO) }\end{array}$ & $\begin{array}{l}\text { Site } \\
\text { Investigator }\end{array}$ & $\begin{array}{l}\text { Data } \\
\text { collection }\end{array}$ \\
\hline $\begin{array}{l}\text { Chiara Rosa } \\
\text { Mancinelli }\end{array}$ & $\begin{array}{l}\text { Centro Sclerosi Multipla } \\
\text { ASST Spedali Civili di } \\
\text { Brescia, Ospedale di } \\
\text { Montichiari }\end{array}$ & $\begin{array}{l}\text { Site } \\
\text { Investigator }\end{array}$ & $\begin{array}{l}\text { Data } \\
\text { collection }\end{array}$ \\
\hline $\begin{array}{l}\text { Luca } \\
\text { Mancinelli }\end{array}$ & $\begin{array}{l}\text { Local Health Agency of } \\
\text { Romagna, Maurizio Bufalini } \\
\text { Hospital (Cesena) - } \\
\text { Neurology Unit }\end{array}$ & $\begin{array}{l}\text { Site } \\
\text { Investigator }\end{array}$ & $\begin{array}{l}\text { Data } \\
\text { collection }\end{array}$ \\
\hline $\begin{array}{l}\text { Paolo } \\
\text { Manganotti }\end{array}$ & $\begin{array}{l}\text { Neurology Unit, } \\
\text { Department of Medical, } \\
\text { Surgical, and Health } \\
\text { Sciences, Cattinara } \\
\text { University Hospital, ASUGI, } \\
\text { Trieste }\end{array}$ & $\begin{array}{l}\text { Site } \\
\text { Investigator }\end{array}$ & $\begin{array}{l}\text { Data } \\
\text { collection }\end{array}$ \\
\hline \multirow[t]{2}{*}{$\begin{array}{l}\text { Giorgia Teresa } \\
\text { Maniscalco }\end{array}$} & $\begin{array}{l}\text { Multiple Sclerosis Centre } \\
\text { "A. Cardarelli Hospital", } \\
\text { Naples, Italy }\end{array}$ & $\begin{array}{l}\text { Site } \\
\text { Investigator }\end{array}$ & $\begin{array}{l}\text { Data } \\
\text { collection }\end{array}$ \\
\hline & $\begin{array}{l}\text { Neurological Clinic and } \\
\text { Stroke Unit "A. Cardarelli } \\
\text { Hospital", Naples, Italy; }\end{array}$ & $\begin{array}{l}\text { Site } \\
\text { Investigator }\end{array}$ & $\begin{array}{l}\text { Data } \\
\text { collection }\end{array}$ \\
\hline $\begin{array}{l}\text { Vittorio } \\
\text { Mantero }\end{array}$ & MS Center, ASST Lecco & $\begin{array}{l}\text { Site } \\
\text { Investigator }\end{array}$ & $\begin{array}{l}\text { Data } \\
\text { collection }\end{array}$ \\
\hline $\begin{array}{l}\text { Sabrina } \\
\text { Marangoni }\end{array}$ & $\begin{array}{l}\text { Ospedale Santa Chiara, } \\
\text { Trento. UO Neurologia } \\
\text { (Trento) }\end{array}$ & $\begin{array}{l}\text { Site } \\
\text { Investigator }\end{array}$ & $\begin{array}{l}\text { Data } \\
\text { collection }\end{array}$ \\
\hline $\begin{array}{l}\text { Damiano } \\
\text { Marastoni }\end{array}$ & $\begin{array}{l}\text { The Multiple Sclerosis } \\
\text { Center of University } \\
\text { Hospital of Verona Dept. of } \\
\text { Neuroscience, } \\
\text { Biomedicine and } \\
\text { Movements }\end{array}$ & $\begin{array}{l}\text { Site } \\
\text { Investigator }\end{array}$ & $\begin{array}{l}\text { Data } \\
\text { collection }\end{array}$ \\
\hline
\end{tabular}

Appendix 2 (continued)

\begin{tabular}{|c|c|c|c|}
\hline Name & Location & Role & Contribution \\
\hline \multirow[t]{2}{*}{$\begin{array}{l}\text { Fabiana } \\
\text { Marinelli }\end{array}$} & $\begin{array}{l}\text { Ospedale Fabrizio } \\
\text { Spaziani Frosinone }\end{array}$ & $\begin{array}{l}\text { Site } \\
\text { Investigator }\end{array}$ & $\begin{array}{l}\text { Data } \\
\text { collection }\end{array}$ \\
\hline & Asl Frosinone & $\begin{array}{l}\text { Site } \\
\text { Investigator }\end{array}$ & $\begin{array}{l}\text { Data } \\
\text { collection }\end{array}$ \\
\hline $\begin{array}{l}\text { Alessandro } \\
\text { Marti }\end{array}$ & $\begin{array}{l}\text { UOC Neurologia- centro } \\
\text { SM Reggio Emilia- AUSL- } \\
\text { IRCSS RE }\end{array}$ & $\begin{array}{l}\text { Site } \\
\text { Investigator }\end{array}$ & $\begin{array}{l}\text { Data } \\
\text { collection }\end{array}$ \\
\hline \multirow[t]{2}{*}{$\begin{array}{l}\text { Filippo } \\
\text { Martinelli } \\
\text { Boneschi }\end{array}$} & $\begin{array}{l}\text { 1. IRCCS Fondazione Ca' } \\
\text { Granda Ospedale } \\
\text { Maggiore Policlinico, } \\
\text { Neurology Unit, Milan, } \\
\text { Italy. Via Francesco Sforza } \\
\text { 35, } 20122\end{array}$ & $\begin{array}{l}\text { Site } \\
\text { Investigator }\end{array}$ & $\begin{array}{l}\text { Data } \\
\text { collection }\end{array}$ \\
\hline & $\begin{array}{l}\text { 2. Dino Ferrari Center, } \\
\text { Department of } \\
\text { Pathophysiology and } \\
\text { Transplantation, } \\
\text { University of Milan, } \\
\text { Milan, Italy. Via Francesco } \\
\text { Sforza 35, } 20122\end{array}$ & $\begin{array}{l}\text { Site } \\
\text { Investigator }\end{array}$ & $\begin{array}{l}\text { Data } \\
\text { collection }\end{array}$ \\
\hline $\begin{array}{l}\text { Federco } \\
\text { Masserano Zoli }\end{array}$ & $\begin{array}{l}\text { AISM Padova } \\
\text { Rehabilitation Service }\end{array}$ & $\begin{array}{l}\text { Site } \\
\text { Investigator }\end{array}$ & $\begin{array}{l}\text { Data } \\
\text { collection }\end{array}$ \\
\hline $\begin{array}{l}\text { Francesca } \\
\text { Matta }\end{array}$ & $\begin{array}{l}\text { Centro SM - UOC } \\
\text { Neurologia - ARNAS } \\
\text { Garibaldi - Catania }\end{array}$ & $\begin{array}{l}\text { Site } \\
\text { Investigator }\end{array}$ & $\begin{array}{l}\text { Data } \\
\text { collection }\end{array}$ \\
\hline $\begin{array}{l}\text { Laura } \\
\text { Mendozzi }\end{array}$ & $\begin{array}{l}\text { IRCCS Fondazione Don } \\
\text { Carlo Gnocchi ONLUS, } \\
\text { Milano }\end{array}$ & $\begin{array}{l}\text { Site } \\
\text { Investigator }\end{array}$ & $\begin{array}{l}\text { Data } \\
\text { collection }\end{array}$ \\
\hline $\begin{array}{l}\text { Giuseppe } \\
\text { Meucci }\end{array}$ & UO Neurologia Livorno & $\begin{array}{l}\text { Site } \\
\text { Investigator }\end{array}$ & $\begin{array}{l}\text { Data } \\
\text { collection }\end{array}$ \\
\hline Silvia Miante & $\begin{array}{l}\text { Multiple Sclerosis Centre } \\
\text { of the Veneto Region } \\
\text { (CeSMuV), University } \\
\text { Hospital of Padua, Italy. }\end{array}$ & $\begin{array}{l}\text { Site } \\
\text { Investigator }\end{array}$ & $\begin{array}{l}\text { Data } \\
\text { collection }\end{array}$ \\
\hline $\begin{array}{l}\text { Giuseppina } \\
\text { Miele }\end{array}$ & $\begin{array}{l}\text { Department of Advanced } \\
\text { Medical and Surgical } \\
\text { Sciences, University of } \\
\text { Campania Luigi Vanvitelli, } \\
80138 \text { Naples, Italy; }\end{array}$ & $\begin{array}{l}\text { Site } \\
\text { Investigator }\end{array}$ & $\begin{array}{l}\text { Data } \\
\text { collection }\end{array}$ \\
\hline Eva Milano & $\begin{array}{l}\text { SC Neurologia1 Ospedale } \\
\text { Maria Vittoria- Torino }\end{array}$ & $\begin{array}{l}\text { Site } \\
\text { Investigator }\end{array}$ & $\begin{array}{l}\text { Data } \\
\text { collection }\end{array}$ \\
\hline $\begin{array}{l}\text { Massimiliano } \\
\text { Mirabella }\end{array}$ & $\begin{array}{l}\text { Fondazione Policlinico } \\
\text { Universitario Agostino } \\
\text { Gemelli IRCCS - } \\
\text { Università Cattolica del } \\
\text { Sacro Cuore }\end{array}$ & $\begin{array}{l}\text { Site } \\
\text { Investigator }\end{array}$ & $\begin{array}{l}\text { Data } \\
\text { collection }\end{array}$ \\
\hline $\begin{array}{l}\text { Rosanna } \\
\text { Missione }\end{array}$ & $\begin{array}{l}\text { II Clinica Neurologica, } \\
\text { Università della } \\
\text { Campania Luigi } \\
\text { Vanvitelli, Naples }\end{array}$ & $\begin{array}{l}\text { Site } \\
\text { Investigator }\end{array}$ & $\begin{array}{l}\text { Data } \\
\text { collection }\end{array}$ \\
\hline $\begin{array}{l}\text { Marcello } \\
\text { Moccia }\end{array}$ & $\begin{array}{l}\text { Federico II University of } \\
\text { Naples }\end{array}$ & $\begin{array}{l}\text { Site } \\
\text { Investigator }\end{array}$ & $\begin{array}{l}\text { Data } \\
\text { collection }\end{array}$ \\
\hline Lucia Moiola & $\begin{array}{l}\text { Neurology Unit, IRCCS } \\
\text { San Raffaele Scientific } \\
\text { Institute, Milan, Italy }\end{array}$ & $\begin{array}{l}\text { Site } \\
\text { Investigator }\end{array}$ & $\begin{array}{l}\text { Data } \\
\text { collection }\end{array}$ \\
\hline $\begin{array}{l}\text { Sara } \\
\text { Montepietra }\end{array}$ & $\begin{array}{l}\text { Responsabile del Centro } \\
\text { Sclerosi Multipla - Reggio } \\
\text { Emilia- UOC Neurologia- } \\
\text { AUSL-IRCSS RE }\end{array}$ & $\begin{array}{l}\text { Site } \\
\text { Investigator }\end{array}$ & $\begin{array}{l}\text { Data } \\
\text { collection }\end{array}$ \\
\hline
\end{tabular}


Appendix 2 (continued)

\begin{tabular}{|c|c|c|c|}
\hline Name & Location & Role & Contribution \\
\hline $\begin{array}{l}\text { Margherita } \\
\text { MontiBragadin }\end{array}$ & $\begin{array}{l}\text { AISM Rehabilitation } \\
\text { Service Liguria }\end{array}$ & $\begin{array}{l}\text { Site } \\
\text { Investigator }\end{array}$ & $\begin{array}{l}\text { Data } \\
\text { collection }\end{array}$ \\
\hline $\begin{array}{l}\text { Federico } \\
\text { Montini }\end{array}$ & $\begin{array}{l}\text { Neurology Unit, IRCCS } \\
\text { San Raffaele Scientific } \\
\text { Institute, Milan, Italy }\end{array}$ & $\begin{array}{l}\text { Site } \\
\text { Investigator }\end{array}$ & $\begin{array}{l}\text { Data } \\
\text { collection }\end{array}$ \\
\hline Roberta Motta & $\begin{array}{l}\text { AISM Rehabilitation } \\
\text { Service Liguria }\end{array}$ & $\begin{array}{l}\text { Site } \\
\text { Investigator }\end{array}$ & $\begin{array}{l}\text { Data } \\
\text { collection }\end{array}$ \\
\hline \multirow[t]{2}{*}{$\begin{array}{l}\text { Raffaele } \\
\text { Nardone }\end{array}$} & $\begin{array}{l}\text { 1. Paracelsus Medical } \\
\text { University, Department of } \\
\text { Neurology, Salisburgo, AU }\end{array}$ & $\begin{array}{l}\text { Site } \\
\text { Investigator }\end{array}$ & $\begin{array}{l}\text { Data } \\
\text { collection }\end{array}$ \\
\hline & $\begin{array}{l}\text { 2. Department of } \\
\text { Neurology - Franz } \\
\text { Tappeiner Hospital } \\
\text { Meran (BZ), Italy }\end{array}$ & $\begin{array}{l}\text { Site } \\
\text { Investigator }\end{array}$ & $\begin{array}{l}\text { Data } \\
\text { collection }\end{array}$ \\
\hline $\begin{array}{l}\text { Carolina Gabri } \\
\text { Nicoletti }\end{array}$ & $\begin{array}{l}\text { Multiple Sclerosis Clinical } \\
\text { and Research Unit, } \\
\text { Department of Systems } \\
\text { Medicine, Tor Vergata } \\
\text { University, Rome, Italy }\end{array}$ & $\begin{array}{l}\text { Site } \\
\text { Investigator }\end{array}$ & $\begin{array}{l}\text { Data } \\
\text { collection }\end{array}$ \\
\hline \multirow[t]{3}{*}{$\begin{array}{l}\text { Eduardo } \\
\text { Nobile-Orazio }\end{array}$} & $\begin{array}{l}\text { 1)Neuromuscular and } \\
\text { Neuroimmunology } \\
\text { Service, IRCCS } \\
\text { Humanitas Clinical and } \\
\text { Research Institute, } \\
\text { Rozzano, Milan, Italy }\end{array}$ & $\begin{array}{l}\text { Site } \\
\text { Investigator }\end{array}$ & $\begin{array}{l}\text { Data } \\
\text { collection }\end{array}$ \\
\hline & $\begin{array}{l}\text { 2)Department of Medical } \\
\text { Biotechnology and } \\
\text { Translational Medicine, } \\
\text { Milan University, Milan, }\end{array}$ & $\begin{array}{l}\text { Site } \\
\text { Investigator }\end{array}$ & $\begin{array}{l}\text { Data } \\
\text { collection }\end{array}$ \\
\hline & Italy & $\begin{array}{l}\text { Site } \\
\text { Investigator }\end{array}$ & $\begin{array}{l}\text { Data } \\
\text { collection }\end{array}$ \\
\hline $\begin{array}{l}\text { Agostino } \\
\text { Nozzolillo }\end{array}$ & $\begin{array}{l}\text { Neurology Unit, IRCCS } \\
\text { San Raffaele Scientific } \\
\text { Institute, Milan, Italy }\end{array}$ & $\begin{array}{l}\text { Site } \\
\text { Investigator }\end{array}$ & $\begin{array}{l}\text { Data } \\
\text { collection }\end{array}$ \\
\hline Marco Onofrj & $\begin{array}{l}\text { Department of } \\
\text { Neurosciences, Imaging } \\
\text { and Clinical Sciences, } \\
\text { University G. d'Annunzio } \\
\text { of Chieti-Pescara, Chieti, } \\
\text { Italy }\end{array}$ & $\begin{array}{l}\text { Site } \\
\text { Investigator }\end{array}$ & $\begin{array}{l}\text { Data } \\
\text { collection }\end{array}$ \\
\hline $\begin{array}{l}\text { Riccardo } \\
\text { Orlandi }\end{array}$ & $\begin{array}{l}\text { Dipartimento di } \\
\text { Neuroscienze, } \\
\text { Biomedicina e } \\
\text { Movimento, Università di } \\
\text { Verona }\end{array}$ & $\begin{array}{l}\text { Site } \\
\text { Investigator }\end{array}$ & $\begin{array}{l}\text { Data } \\
\text { collection }\end{array}$ \\
\hline Anna Palmieri & UO Neurologia, Treviso & $\begin{array}{l}\text { Site } \\
\text { Investigator }\end{array}$ & $\begin{array}{l}\text { Data } \\
\text { collection }\end{array}$ \\
\hline $\begin{array}{l}\text { Damiano } \\
\text { Paolicelli }\end{array}$ & $\begin{array}{l}\text { Department of Basic } \\
\text { Medical Sciences, } \\
\text { Neurosciences and } \\
\text { Sense Organs - University } \\
\text { of Bari Aldo Moro }\end{array}$ & $\begin{array}{l}\text { Site } \\
\text { Investigator }\end{array}$ & $\begin{array}{l}\text { Data } \\
\text { collection }\end{array}$ \\
\hline Livia Pasquali & $\begin{array}{l}\text { Department of Clinical } \\
\text { and Experimental } \\
\text { Medicine, Neurology } \\
\text { Unit, University of Pisa, } \\
\text { Pisa, Italy }\end{array}$ & $\begin{array}{l}\text { Site } \\
\text { Investigator }\end{array}$ & $\begin{array}{l}\text { Data } \\
\text { collection }\end{array}$ \\
\hline Luisa Pastò & $\begin{array}{l}\text { Azienda Ospedaliero } \\
\text { Universitaria Careggi, } \\
\text { Firenze }\end{array}$ & $\begin{array}{l}\text { Site } \\
\text { Investigator }\end{array}$ & $\begin{array}{l}\text { Data } \\
\text { collection }\end{array}$ \\
\hline
\end{tabular}

Appendix 2 (continued)

\begin{tabular}{|c|c|c|c|}
\hline Name & Location & Role & Contribution \\
\hline $\begin{array}{l}\text { Elisabetta } \\
\text { Pedrazzoli }\end{array}$ & $\begin{array}{l}\text { AISM Padova } \\
\text { Rehabilitation Service }\end{array}$ & $\begin{array}{l}\text { Site } \\
\text { Investigator }\end{array}$ & $\begin{array}{l}\text { Data } \\
\text { collection }\end{array}$ \\
\hline Maria Petracca & $\begin{array}{l}\text { Federico II University of } \\
\text { Naples }\end{array}$ & $\begin{array}{l}\text { Site } \\
\text { Investigator }\end{array}$ & $\begin{array}{l}\text { Data } \\
\text { collection }\end{array}$ \\
\hline $\begin{array}{l}\text { Alfredo } \\
\text { Petrone }\end{array}$ & $\begin{array}{l}\text { Ospedale Annunziata } \\
\text { (Cosenza) }\end{array}$ & $\begin{array}{l}\text { Site } \\
\text { Investigator }\end{array}$ & $\begin{array}{l}\text { Data } \\
\text { collection }\end{array}$ \\
\hline $\begin{array}{l}\text { Carlo } \\
\text { Piantadosi }\end{array}$ & $\begin{array}{l}\text { UOC Neurologia - } \\
\text { Azienda Ospedaliera } \\
\text { "San Giovanni- } \\
\text { Addolorata" - Roma }\end{array}$ & $\begin{array}{l}\text { Site } \\
\text { Investigator }\end{array}$ & $\begin{array}{l}\text { Data } \\
\text { collection }\end{array}$ \\
\hline \multirow[t]{2}{*}{$\begin{array}{l}\text { Anna M. } \\
\text { Pietroboni }\end{array}$} & $\begin{array}{l}\text { 1. Fondazione IRCCS Ca' } \\
\text { Granda Ospedale } \\
\text { Maggiore Policlinico, } \\
\text { Milan, IT. }\end{array}$ & $\begin{array}{l}\text { Site } \\
\text { Investigator }\end{array}$ & $\begin{array}{l}\text { Data } \\
\text { collection }\end{array}$ \\
\hline & $\begin{array}{l}\text { 2. University of Milan, } \\
\text { Dino Ferrari Center, } \\
\text { Milan, IT. }\end{array}$ & $\begin{array}{l}\text { Site } \\
\text { Investigator }\end{array}$ & $\begin{array}{l}\text { Data } \\
\text { collection }\end{array}$ \\
\hline $\begin{array}{l}\text { Federica } \\
\text { Pinardi }\end{array}$ & $\begin{array}{l}\text { IRCCS Istituto delle } \\
\text { Scienze Neurologiche di } \\
\text { Bologna, UOSI } \\
\text { Riabilitazione Sclerosi } \\
\text { Multipla }\end{array}$ & $\begin{array}{l}\text { Site } \\
\text { Investigator }\end{array}$ & $\begin{array}{l}\text { Data } \\
\text { collection }\end{array}$ \\
\hline $\begin{array}{l}\text { Marta } \\
\text { Ponzano }\end{array}$ & $\begin{array}{l}\text { Department of Health } \\
\text { Sciences, University of } \\
\text { Genoa, Genoa, Italy. }\end{array}$ & Biostatistician & $\begin{array}{l}\text { Support in } \\
\text { statistical } \\
\text { analysis }\end{array}$ \\
\hline $\begin{array}{l}\text { Emilio } \\
\text { Portaccio }\end{array}$ & $\begin{array}{l}\text { Università degli Studi di } \\
\text { Firenze, Dipartimento } \\
\text { NEUROFARBA, Firenze }\end{array}$ & $\begin{array}{l}\text { Site } \\
\text { Investigator }\end{array}$ & $\begin{array}{l}\text { Data } \\
\text { collection }\end{array}$ \\
\hline \multirow[t]{2}{*}{ Mattia Pozzato } & $\begin{array}{l}\text { 1. IRCCS Fondazione Ca' } \\
\text { Granda Ospedale } \\
\text { Maggiore Policlinico, } \\
\text { Neurology Unit, } \\
\text { Milan, Italy. Via } \\
\text { Francesco Sforza 35, } \\
20122\end{array}$ & $\begin{array}{l}\text { Site } \\
\text { Investigator }\end{array}$ & $\begin{array}{l}\text { Data } \\
\text { collection }\end{array}$ \\
\hline & $\begin{array}{l}\text { 2. Dino Ferrari Center, } \\
\text { Department of } \\
\text { Pathophysiology and } \\
\text { Transplantation, } \\
\text { University of Milan, } \\
\text { Milan, Italy. Via Francesco } \\
\text { Sforza 35, } 20122\end{array}$ & $\begin{array}{l}\text { Site } \\
\text { Investigator }\end{array}$ & $\begin{array}{l}\text { Data } \\
\text { collection }\end{array}$ \\
\hline Carlo Pozzilli & $\begin{array}{l}\text { Centro SM S.Andrea } \\
\text { Dip.Neuroscienze } \\
\text { Umane Sapienza Roma }\end{array}$ & $\begin{array}{l}\text { Site } \\
\text { Investigator }\end{array}$ & $\begin{array}{l}\text { Data } \\
\text { collection }\end{array}$ \\
\hline $\begin{array}{l}\text { Luca } \\
\text { Prosperini }\end{array}$ & $\begin{array}{l}\text { Dept Neurosciences, San } \\
\text { Camillo Forlanini } \\
\text { Hospital - Rome }\end{array}$ & $\begin{array}{l}\text { Site } \\
\text { Investigator }\end{array}$ & $\begin{array}{l}\text { Data } \\
\text { collection }\end{array}$ \\
\hline $\begin{array}{l}\text { Alessandra } \\
\text { Protti }\end{array}$ & $\begin{array}{l}\text { ASST GOM NIGUARDA, } \\
\text { DIPARTIMENTO } \\
\text { NEUROSCIENZE }\end{array}$ & $\begin{array}{l}\text { Site } \\
\text { Investigator }\end{array}$ & $\begin{array}{l}\text { Data } \\
\text { collection }\end{array}$ \\
\hline $\begin{array}{l}\text { Paolo } \\
\text { Ragonese }\end{array}$ & $\begin{array}{l}\text { Department of } \\
\text { Biomedicine, } \\
\text { Neurosciences and } \\
\text { Advanced Diagnsotics; } \\
\text { University of Palermo }\end{array}$ & $\begin{array}{l}\text { Site } \\
\text { Investigator }\end{array}$ & $\begin{array}{l}\text { Data } \\
\text { collection }\end{array}$ \\
\hline Sarah Rasia & $\begin{array}{l}\text { Centro Sclerosi Multipla } \\
\text { ASST Spedali Civili di } \\
\text { Brescia, Ospedale di } \\
\text { Montichiari }\end{array}$ & $\begin{array}{l}\text { Site } \\
\text { Investigator }\end{array}$ & $\begin{array}{l}\text { Data } \\
\text { collection }\end{array}$ \\
\hline
\end{tabular}


Appendix 2 (continued)

\begin{tabular}{|c|c|c|c|}
\hline Name & Location & Role & Contribution \\
\hline $\begin{array}{l}\text { Sabrina } \\
\text { Realmuto }\end{array}$ & $\begin{array}{l}\text { Centro Sclerosi Multipla, } \\
\text { UOC di Neurologia e } \\
\text { Stroke Unit, AOOR Villa } \\
\text { Sofia-Cervello Palermo }\end{array}$ & $\begin{array}{l}\text { Site } \\
\text { Investigator }\end{array}$ & $\begin{array}{l}\text { Data } \\
\text { collection }\end{array}$ \\
\hline Anna Repice & $\begin{array}{l}\text { Azienda Ospedaliera } \\
\text { Universitaria Careggi } \\
\text { Firenze }\end{array}$ & $\begin{array}{l}\text { Site } \\
\text { Investigator }\end{array}$ & $\begin{array}{l}\text { Data } \\
\text { collection }\end{array}$ \\
\hline $\begin{array}{l}\text { Eleonora } \\
\text { Rigoni }\end{array}$ & $\begin{array}{l}\text { IRCCS Mondino } \\
\text { Foundation, Pavia }\end{array}$ & $\begin{array}{l}\text { Site } \\
\text { Investigator }\end{array}$ & $\begin{array}{l}\text { Data } \\
\text { collection }\end{array}$ \\
\hline $\begin{array}{l}\text { Maria Teresa } \\
\text { Rilla }\end{array}$ & $\begin{array}{l}\text { Neurologia ASL } 1 \\
\text { imperiese }\end{array}$ & $\begin{array}{l}\text { Site } \\
\text { Investigator }\end{array}$ & $\begin{array}{l}\text { Data } \\
\text { collection }\end{array}$ \\
\hline $\begin{array}{l}\text { Francesca } \\
\text { Rinaldi }\end{array}$ & $\begin{array}{l}\text { Multiple Sclerosis Centre } \\
\text { of the Veneto Region } \\
\text { (CeSMuV), University } \\
\text { Hospital of Padua, Italy. }\end{array}$ & $\begin{array}{l}\text { Site } \\
\text { Investigator }\end{array}$ & $\begin{array}{l}\text { Data } \\
\text { collection }\end{array}$ \\
\hline $\begin{array}{l}\text { Calogero } \\
\text { Marcello } \\
\text { Romano }\end{array}$ & $\begin{array}{l}\text { Centro Sclerosi Multipla, } \\
\text { UOC di Neurologia e } \\
\text { Stroke Unit, AOOR Villa } \\
\text { Sofia-Cervello Palermo }\end{array}$ & $\begin{array}{l}\text { Site } \\
\text { Investigator }\end{array}$ & $\begin{array}{l}\text { Data } \\
\text { collection }\end{array}$ \\
\hline Marco Ronzoni & ASST Rhodense & $\begin{array}{l}\text { Site } \\
\text { Investigator }\end{array}$ & $\begin{array}{l}\text { Data } \\
\text { collection }\end{array}$ \\
\hline Marco Rovaris & $\begin{array}{l}\text { IRCCS Fondazione Don } \\
\text { Carlo Gnocchi ONLUS, } \\
\text { Milano }\end{array}$ & $\begin{array}{l}\text { Site } \\
\text { Investigator }\end{array}$ & $\begin{array}{l}\text { Data } \\
\text { collection }\end{array}$ \\
\hline $\begin{array}{l}\text { Francesca } \\
\text { Ruscica }\end{array}$ & $\begin{array}{l}\text { centro SM reparto } \\
\text { neurologia Ospedale G. } \\
\text { Giglio, Cefalù (PA) }\end{array}$ & $\begin{array}{l}\text { Site } \\
\text { Investigator }\end{array}$ & $\begin{array}{l}\text { Data } \\
\text { collection }\end{array}$ \\
\hline $\begin{array}{l}\text { Loredana } \\
\text { Sabattini }\end{array}$ & $\begin{array}{l}\text { IRCCS Istituto delle } \\
\text { Scienze Neurologiche di } \\
\text { Bologna, UOSI } \\
\text { Riabilitazione Sclerosi } \\
\text { Multipla }\end{array}$ & $\begin{array}{l}\text { Site } \\
\text { Investigator }\end{array}$ & $\begin{array}{l}\text { Data } \\
\text { collection }\end{array}$ \\
\hline $\begin{array}{l}\text { Giuseppe } \\
\text { Salemi }\end{array}$ & $\begin{array}{l}\text { Department of } \\
\text { Biomedicine, } \\
\text { Neurosciences and } \\
\text { advanced Diagnostics; } \\
\text { University of Palermo }\end{array}$ & $\begin{array}{l}\text { Site } \\
\text { Investigator }\end{array}$ & $\begin{array}{l}\text { Data } \\
\text { collection }\end{array}$ \\
\hline $\begin{array}{l}\text { Lorenzo } \\
\text { Saraceno }\end{array}$ & $\begin{array}{l}\text { ASST GOM NIGUARDA, } \\
\text { DIPARTIMENTO } \\
\text { NEUROSCIENZE }\end{array}$ & $\begin{array}{l}\text { Site } \\
\text { Investigator }\end{array}$ & $\begin{array}{l}\text { Data } \\
\text { collection }\end{array}$ \\
\hline Alessia Sartori & $\begin{array}{l}\text { Department of } \\
\text { Pharmacy, Ospedale } \\
\text { Guglielmo da Saliceto, } \\
\text { Piacenza, Italy }\end{array}$ & $\begin{array}{l}\text { Site } \\
\text { Investigator }\end{array}$ & $\begin{array}{l}\text { Data } \\
\text { collection }\end{array}$ \\
\hline $\begin{array}{l}\text { Arianna } \\
\text { Sartori }\end{array}$ & $\begin{array}{l}\text { Neurology Unit, } \\
\text { Department of Medical, } \\
\text { Surgical, and Health } \\
\text { Sciences, Cattinara } \\
\text { University Hospital, } \\
\text { ASUGI, Trieste }\end{array}$ & $\begin{array}{l}\text { Site } \\
\text { Investigator }\end{array}$ & $\begin{array}{l}\text { Data } \\
\text { collection }\end{array}$ \\
\hline Elvira Sbragia & $\begin{array}{l}\text { DINOGMI Universita' di } \\
\text { Genova }\end{array}$ & $\begin{array}{l}\text { Site } \\
\text { Investigator }\end{array}$ & $\begin{array}{l}\text { Data } \\
\text { collection }\end{array}$ \\
\hline \multirow[t]{2}{*}{$\begin{array}{l}\text { Giuditta Ilaria } \\
\text { Scarano }\end{array}$} & $\begin{array}{l}\text { 1. Departement of } \\
\text { Psycology, University of } \\
\text { Milano-Bicocca, Milan, } \\
\text { Italy }\end{array}$ & $\begin{array}{l}\text { Site } \\
\text { Investigator }\end{array}$ & $\begin{array}{l}\text { Data } \\
\text { collection }\end{array}$ \\
\hline & $\begin{array}{l}\text { 2. Departement of } \\
\text { Psychology, Franz } \\
\text { Tappeiner Hospital, } \\
\text { Merano, Italy }\end{array}$ & $\begin{array}{l}\text { Site } \\
\text { Investigator }\end{array}$ & $\begin{array}{l}\text { Data } \\
\text { collection }\end{array}$ \\
\hline
\end{tabular}

Appendix 2 (continued)

\begin{tabular}{|c|c|c|c|}
\hline Name & Location & Role & Contribution \\
\hline $\begin{array}{l}\text { Valentina } \\
\text { Scarano }\end{array}$ & $\begin{array}{l}\text { UOC Neurologia e Stroke } \\
\text { AORN San Giuseppe } \\
\text { Moscati Avellino }\end{array}$ & $\begin{array}{l}\text { Site } \\
\text { Investigator }\end{array}$ & $\begin{array}{l}\text { Data } \\
\text { collection }\end{array}$ \\
\hline $\begin{array}{l}\text { Valentina } \\
\text { Schillaci }\end{array}$ & $\begin{array}{l}\text { MS Center, Department } \\
\text { of Neuroscience, City of } \\
\text { Health and Science } \\
\text { University Hospital of } \\
\text { Turin, Turin, Italy }\end{array}$ & $\begin{array}{l}\text { Site } \\
\text { Investigator }\end{array}$ & $\begin{array}{l}\text { Data } \\
\text { collection }\end{array}$ \\
\hline Maria Sessa & $\begin{array}{l}\text { UOC Neurologia, USS } \\
\text { Neuroimmunologia, } \\
\text { ASST Papa Giovanni XXIII }\end{array}$ & $\begin{array}{l}\text { Site } \\
\text { Investigator }\end{array}$ & $\begin{array}{l}\text { Data } \\
\text { collection }\end{array}$ \\
\hline $\begin{array}{l}\text { Caterina } \\
\text { Sgarito }\end{array}$ & $\begin{array}{l}\text { AISM Como } \\
\text { Rehabilitation Service }\end{array}$ & $\begin{array}{l}\text { Site } \\
\text { Investigator }\end{array}$ & $\begin{array}{l}\text { Data } \\
\text { collection }\end{array}$ \\
\hline Grazia Sibilia & $\begin{array}{l}\text { NEUROLOGY UNIT AND } \\
\text { MS CENTER, ASL NAPOLI } \\
1 \text { CENTRO, NAPLES, ITALY }\end{array}$ & $\begin{array}{l}\text { Site } \\
\text { Investigator }\end{array}$ & $\begin{array}{l}\text { Data } \\
\text { collection }\end{array}$ \\
\hline $\begin{array}{l}\text { Gabriele } \\
\text { Siciliano }\end{array}$ & $\begin{array}{l}\text { Department of Clinical } \\
\text { and Experimental } \\
\text { Medicine, Neurology } \\
\text { Unit, University of Pisa, } \\
\text { Pisa, Italy }\end{array}$ & $\begin{array}{l}\text { Site } \\
\text { Investigator }\end{array}$ & $\begin{array}{l}\text { Data } \\
\text { collection }\end{array}$ \\
\hline Alessio Signori & $\begin{array}{l}\text { Department of Health } \\
\text { Sciences, University of } \\
\text { Genoa, Genoa, Italy. }\end{array}$ & Biostatistician & $\begin{array}{l}\text { Support in } \\
\text { statistical } \\
\text { analysis }\end{array}$ \\
\hline $\begin{array}{l}\text { Elisabetta } \\
\text { Signoriello }\end{array}$ & $\begin{array}{l}\text { Il Clinica Neurologica, } \\
\text { Università della } \\
\text { Campania Luigi } \\
\text { Vanvitelli, Naples }\end{array}$ & $\begin{array}{l}\text { Site } \\
\text { Investigator }\end{array}$ & $\begin{array}{l}\text { Data } \\
\text { collection }\end{array}$ \\
\hline $\begin{array}{l}\text { Leonardo } \\
\text { Sinisi }\end{array}$ & $\begin{array}{l}\text { NEUROLOGY UNIT AND } \\
\text { MS CENTER, ASL } \\
\text { NAPOLI } 1 \text { CENTRO, } \\
\text { NAPLES, ITALY }\end{array}$ & $\begin{array}{l}\text { Site } \\
\text { Investigator }\end{array}$ & $\begin{array}{l}\text { Data } \\
\text { collection }\end{array}$ \\
\hline $\begin{array}{l}\text { Francesca } \\
\text { Sireci }\end{array}$ & $\begin{array}{l}\text { UOC Neurologia- centro } \\
\text { SM Reggio Emilia- AUSL- } \\
\text { IRCCS RE }\end{array}$ & $\begin{array}{l}\text { Site } \\
\text { Investigator }\end{array}$ & $\begin{array}{l}\text { Data } \\
\text { collection }\end{array}$ \\
\hline Patrizia Sola & $\begin{array}{l}\text { Neurology Unit, } \\
\text { Ospedale Civile, Azienda } \\
\text { Ospedaliero- } \\
\text { Universitaria di Modena, } \\
\text { Modena, Italy }\end{array}$ & $\begin{array}{l}\text { Site } \\
\text { Investigator }\end{array}$ & $\begin{array}{l}\text { Data } \\
\text { collection }\end{array}$ \\
\hline Claudio Solaro & $\begin{array}{l}\text { Dept. of Rehabilitation, } \\
\text { CRRF "Mons. Luigi } \\
\text { Novarese", Moncrivello, } \\
\text { Italy }\end{array}$ & $\begin{array}{l}\text { Site } \\
\text { Investigator }\end{array}$ & $\begin{array}{l}\text { Data } \\
\text { collection }\end{array}$ \\
\hline Stefano Sotgiu & $\begin{array}{l}\text { Dipartimento di Scienze } \\
\text { Mediche, Chirurgiche e } \\
\text { Sperimentali - Università } \\
\text { di Sassari }\end{array}$ & $\begin{array}{l}\text { Site } \\
\text { Investigator }\end{array}$ & $\begin{array}{l}\text { Data } \\
\text { collection }\end{array}$ \\
\hline $\begin{array}{l}\text { Maddalena } \\
\text { Sparaco }\end{array}$ & $\begin{array}{l}\text { Department of Advanced } \\
\text { Medical and Surgical } \\
\text { Sciences, University of } \\
\text { Campania Luigi } \\
\text { Vanvitelli, } 80138 \text { Naples, } \\
\text { Italy; }\end{array}$ & $\begin{array}{l}\text { Site } \\
\text { Investigator }\end{array}$ & $\begin{array}{l}\text { Data } \\
\text { collection }\end{array}$ \\
\hline $\begin{array}{l}\text { Maria Laura } \\
\text { Stromillo }\end{array}$ & $\begin{array}{l}\text { Department of Medicine, } \\
\text { Surgery and } \\
\text { Neuroscience, University } \\
\text { of Siena, Italy }\end{array}$ & $\begin{array}{l}\text { Site } \\
\text { Investigator }\end{array}$ & $\begin{array}{l}\text { Data } \\
\text { collection }\end{array}$ \\
\hline Silvia Strumia & $\begin{array}{l}\text { MS center, Neurology } \\
\text { Unit, Morgagni- } \\
\text { Pierantoni Hospital, Forli }\end{array}$ & $\begin{array}{l}\text { Site } \\
\text { Investigator }\end{array}$ & $\begin{array}{l}\text { Data } \\
\text { collection }\end{array}$ \\
\hline
\end{tabular}


Appendix 2 (continued)

\begin{tabular}{|c|c|c|c|}
\hline Name & Location & Role & Contribution \\
\hline $\begin{array}{l}\text { Emanuela } \\
\text { Laura Susani }\end{array}$ & $\begin{array}{l}\text { ASST GOM NIGUARDA, } \\
\text { DIPARTIMENTO } \\
\text { NEUROSCIENZE }\end{array}$ & $\begin{array}{l}\text { Site } \\
\text { Investigator }\end{array}$ & $\begin{array}{l}\text { Data } \\
\text { collection }\end{array}$ \\
\hline $\begin{array}{l}\text { Giulietta } \\
\text { Tabiadon }\end{array}$ & $\begin{array}{l}\text { Multiple Sclerosis } \\
\text { Outpt.Clinic, Clinical } \\
\text { Neurology and Stroke } \\
\text { Unit Dep., Central } \\
\text { Country Hospital, } \\
\text { Bolzano, Italy }\end{array}$ & $\begin{array}{l}\text { Site } \\
\text { Investigator }\end{array}$ & $\begin{array}{l}\text { Data } \\
\text { collection }\end{array}$ \\
\hline $\begin{array}{l}\text { Francesco } \\
\text { Teatini }\end{array}$ & $\begin{array}{l}\text { Multiple Sclerosis } \\
\text { Outpt.Clinic, Clinical } \\
\text { Neurology and Stroke } \\
\text { Unit Dep., Central } \\
\text { Country Hospital, } \\
\text { Bolzano, Italy }\end{array}$ & $\begin{array}{l}\text { Site } \\
\text { Investigator }\end{array}$ & $\begin{array}{l}\text { Data } \\
\text { collection }\end{array}$ \\
\hline \multirow[t]{2}{*}{$\begin{array}{l}\text { Valentina } \\
\text { Tomassini }\end{array}$} & $\begin{array}{l}\text { 1. Institute for Advanced } \\
\text { Biomedical } \\
\text { Technologies (ITAB), } \\
\text { Department of } \\
\text { Neurosciences, Imaging } \\
\text { and Clinical Sciences, } \\
\text { University G. d'Annunzio } \\
\text { of Chieti-Pescara, Chieti, } \\
\text { Italy }\end{array}$ & $\begin{array}{l}\text { Site } \\
\text { Investigator }\end{array}$ & $\begin{array}{l}\text { Data } \\
\text { collection }\end{array}$ \\
\hline & $\begin{array}{l}\text { 2. MS Centre, } \\
\text { Department of Clinical } \\
\text { Neurology, SS. } \\
\text { Annunziata University } \\
\text { Hospital, Chieti, Italy }\end{array}$ & $\begin{array}{l}\text { Site } \\
\text { Investigator }\end{array}$ & $\begin{array}{l}\text { Data } \\
\text { collection }\end{array}$ \\
\hline $\begin{array}{l}\text { Simone } \\
\text { Tonietti }\end{array}$ & $\begin{array}{l}\text { Centro Sclerosi Multipla } \\
\text { Ospedale San Carlo } \\
\text { Borromeo (Milano) }\end{array}$ & $\begin{array}{l}\text { Site } \\
\text { Investigator }\end{array}$ & $\begin{array}{l}\text { Data } \\
\text { collection }\end{array}$ \\
\hline $\begin{array}{l}\text { Valentina Torri } \\
\text { Clerici }\end{array}$ & $\begin{array}{l}\text { Centro Sclerosi Multipla, } \\
\text { U.O Neurologia IV, } \\
\text { Fondazione IRCCS } \\
\text { Istituto Neurologico } \\
\text { "Carlo Besta", Milano }\end{array}$ & $\begin{array}{l}\text { Site } \\
\text { Investigator }\end{array}$ & $\begin{array}{l}\text { Data } \\
\text { collection }\end{array}$ \\
\hline $\begin{array}{l}\text { Carla } \\
\text { Tortorella }\end{array}$ & $\begin{array}{l}\text { Dept Neurosciences, San } \\
\text { Camillo Forlanini } \\
\text { Hospital - Rome }\end{array}$ & $\begin{array}{l}\text { Site } \\
\text { Investigator }\end{array}$ & $\begin{array}{l}\text { Data } \\
\text { collection }\end{array}$ \\
\hline $\begin{array}{l}\text { Simona } \\
\text { Toscano }\end{array}$ & $\begin{array}{l}\text { Dipartimento Scienze } \\
\text { Mediche e Chirurgiche e } \\
\text { Tecnologie Avanzate, GF } \\
\text { Ingrassia, Università di } \\
\text { Catania; Centro Sclerosi } \\
\text { Multipla Policlinico "G } \\
\text { Rodolico"- San Marco, } \\
\text { Università di Catania }\end{array}$ & $\begin{array}{l}\text { Site } \\
\text { Investigator }\end{array}$ & $\begin{array}{l}\text { Data } \\
\text { collection }\end{array}$ \\
\hline Rocco Totaro & $\begin{array}{l}\text { Demyelinating Disease } \\
\text { Center, San Salvatore } \\
\text { Hospital, L'Aquila }\end{array}$ & $\begin{array}{l}\text { Site } \\
\text { Investigator }\end{array}$ & $\begin{array}{l}\text { Data } \\
\text { collection }\end{array}$ \\
\hline Maria Trotta & $\begin{array}{l}\text { Ospedale Annunziata } \\
\text { (Cosenza) }\end{array}$ & $\begin{array}{l}\text { Site } \\
\text { Investigator }\end{array}$ & $\begin{array}{l}\text { Data } \\
\text { collection }\end{array}$ \\
\hline $\begin{array}{l}\text { Gabriella } \\
\text { Turano }\end{array}$ & $\begin{array}{l}\text { Department of } \\
\text { Neurology, Regina } \\
\text { Montis Regalis Hospital, } \\
\text { Mondovi (CN) }\end{array}$ & $\begin{array}{l}\text { Site } \\
\text { Investigator }\end{array}$ & $\begin{array}{l}\text { Data } \\
\text { collection }\end{array}$ \\
\hline Monica Ulivelli & $\begin{array}{l}\text { Department of Medicine, } \\
\text { Surgery and } \\
\text { Neuroscience, University } \\
\text { of Siena, Siena, Italy }\end{array}$ & $\begin{array}{l}\text { Site } \\
\text { Investigator }\end{array}$ & $\begin{array}{l}\text { Data } \\
\text { collection }\end{array}$ \\
\hline
\end{tabular}

Appendix 2 (continued)

\begin{tabular}{|c|c|c|c|}
\hline Name & Location & Role & Contribution \\
\hline $\begin{array}{l}\text { Manzo } \\
\text { Valentino }\end{array}$ & $\begin{array}{l}\text { Neurological Clinic and } \\
\text { Stroke Unit "A. Cardarelli } \\
\text { Hospital", Naples, Italy; }\end{array}$ & $\begin{array}{l}\text { Site } \\
\text { Investigator }\end{array}$ & $\begin{array}{l}\text { Data } \\
\text { collection }\end{array}$ \\
\hline $\begin{array}{l}\text { Giovanna } \\
\text { Vaula }\end{array}$ & $\begin{array}{l}\text { Neurology II, } \\
\text { AOU Città della } \\
\text { Salute e della Scienza } \\
\text {-Turin }\end{array}$ & $\begin{array}{l}\text { Site } \\
\text { Investigator }\end{array}$ & $\begin{array}{l}\text { Data } \\
\text { collection }\end{array}$ \\
\hline $\begin{array}{l}\text { Domizia } \\
\text { Vecchio }\end{array}$ & $\begin{array}{l}\text { Neurology Unit, } \\
\text { Maggiore della Carità } \\
\text { Hospital, Department } \\
\text { of Translational } \\
\text { Medicine, University of } \\
\text { Piemonte Orientale, } \\
\text { Novara, Italy }\end{array}$ & $\begin{array}{l}\text { Site } \\
\text { Investigator }\end{array}$ & $\begin{array}{l}\text { Data } \\
\text { collection }\end{array}$ \\
\hline $\begin{array}{l}\text { Marco } \\
\text { Vercellino }\end{array}$ & $\begin{array}{l}\text { MS Center, Department } \\
\text { of Neuroscience, City of } \\
\text { Health and Science } \\
\text { University Hospital of } \\
\text { Turin, Turin, Italy }\end{array}$ & $\begin{array}{l}\text { Site } \\
\text { Investigator }\end{array}$ & $\begin{array}{l}\text { Data } \\
\text { collection }\end{array}$ \\
\hline $\begin{array}{l}\text { Elena Pinuccia } \\
\text { Verrengia }\end{array}$ & $\begin{array}{l}\text { Centro Sclerosi Multipla, } \\
\text { Ospedale di Legnano, } \\
\text { ASST OVEST MI, Italia }\end{array}$ & $\begin{array}{l}\text { Site } \\
\text { Investigator }\end{array}$ & $\begin{array}{l}\text { Data } \\
\text { collection }\end{array}$ \\
\hline $\begin{array}{l}\text { Marika } \\
\text { Vianello }\end{array}$ & UO Neurologia, Treviso & $\begin{array}{l}\text { Site } \\
\text { Investigator }\end{array}$ & $\begin{array}{l}\text { Data } \\
\text { collection }\end{array}$ \\
\hline $\begin{array}{l}\text { Eleonora } \\
\text { Virgilio }\end{array}$ & $\begin{array}{l}\text { Neurology Unit, } \\
\text { Maggiore della Carità } \\
\text { Hospital, Department of } \\
\text { Translational Medicine, } \\
\text { University of Piemonte } \\
\text { Orientale, Novara, Italy }\end{array}$ & $\begin{array}{l}\text { Site } \\
\text { Investigator }\end{array}$ & $\begin{array}{l}\text { Data } \\
\text { collection }\end{array}$ \\
\hline $\begin{array}{l}\text { Francesca } \\
\text { Vitetta }\end{array}$ & $\begin{array}{l}\text { Neurology Unit, } \\
\text { Ospedale Civile, Azienda } \\
\text { Ospedaliero- } \\
\text { Universitaria di Modena, } \\
\text { Modena, Italy }\end{array}$ & $\begin{array}{l}\text { Site } \\
\text { Investigator }\end{array}$ & $\begin{array}{l}\text { Data } \\
\text { collection }\end{array}$ \\
\hline $\begin{array}{l}\text { Stefano } \\
\text { Vollaro }\end{array}$ & $\begin{array}{l}\text { Emergency Department, } \\
\text { Guglielmo da Saliceto } \\
\text { Hospital, Piacenza, Italy }\end{array}$ & $\begin{array}{l}\text { Site } \\
\text { Investigator }\end{array}$ & $\begin{array}{l}\text { Data } \\
\text { collection }\end{array}$ \\
\hline $\begin{array}{l}\text { Mauro } \\
\text { Zaffaroni }\end{array}$ & $\begin{array}{l}\text { Centro Sclerosi Multipla } \\
\text { Ospedale di Gallarate, } \\
\text { ASST della Valle Olona }\end{array}$ & $\begin{array}{l}\text { Site } \\
\text { Investigator }\end{array}$ & $\begin{array}{l}\text { Data } \\
\text { collection }\end{array}$ \\
\hline $\begin{array}{l}\text { Mauro } \\
\text { Zampolini }\end{array}$ & $\begin{array}{l}\text { Neurologia, Ospedale } \\
\text { San Giovanni, Foligno }\end{array}$ & $\begin{array}{l}\text { Site } \\
\text { Investigator }\end{array}$ & $\begin{array}{l}\text { Data } \\
\text { collection }\end{array}$ \\
\hline $\begin{array}{l}\text { Ignazio } \\
\text { Roberto Zarbo }\end{array}$ & $\begin{array}{l}\text { Dipartimento di Scienze } \\
\text { Mediche, Chirurgiche e } \\
\text { Sperimentali - Università } \\
\text { di Sassari }\end{array}$ & $\begin{array}{l}\text { Site } \\
\text { Investigator }\end{array}$ & $\begin{array}{l}\text { Data } \\
\text { collection }\end{array}$ \\
\hline Luigi Zuliani & $\begin{array}{l}\text { Department of } \\
\text { Neurology - Ospedale } \\
\text { San Bortolo - AULSS8 } \\
\text { Berica Vicenza }\end{array}$ & & $\begin{array}{l}\text { Data } \\
\text { collection }\end{array}$ \\
\hline
\end{tabular}

\section{References}

1. Sormani MP, De Rossi N, Schiavetti I, et al. Disease-modifying therapies and coronavirus disease 2019 severity in multiple sclerosis. Ann Neurol 2021;89(4):780-789. doi: 10.1002/ana.26028.

2. Louapre C, Collongues N, Stankoff B, et al. Clinical characteristics and outcomes in patients with coronavirus disease 2019 and multiple sclerosis. JAMA Neurol 2020; 77(9):1079-1088. doi: 10.1001/jamaneurol.2020.2581. 
3. Salter A, Fox RJ, Newsome SD, et al. Outcomes and risk factors associated with SARS-CoV-2 infection in a North American registry of patients with multiple sclerosis. JAMA Neurol 2021;78(6):699-708. doi: 10.1001/jamaneurol.2021. 0688 .

4. Sormani MP, Salvetti M, Labauge P, et al. DMTs and Covid-19 severity in MS: a pooled analysis from Italy and France. Ann Clin Transl Neurol 2021;8(8):1738-1744. doi: $10.1002 / \mathrm{acn} 3.51408$.

5. Barzegar M, Mirmosayyeb O, Gajarzadeh M, et al. COVID-19 among patients with multiple sclerosis: a systematic review. Neurol Neuroimmunol Neuroinflammation 2021;8(4):e1001. doi: 10.1212/NXI.0000000000001001.

6. Salvetti M, Bellucci G, Ballerini C, et al. SARS-CoV-2 meta-interactome suggests disease-specific, autoimmune pathophysiologies and therapeutic targets. F1000Res 2020;9:992. doi: 10.12688/f1000research.25593.1.

7. Wang EY, Mao T, Klein J, et al. Diverse functional autoantibodies in patients with COVID-19. Nature 2021;595(7866):283-288. doi: 10.1038/s41586-021-03631-y.
8. Bastard P, Orlova E, Sozaeva L, et al. Preexisting autoantibodies to type I IFNs underlie critical COVID-19 pneumonia in patients with APS-1. J Exp Med 2021; 218(7):e20210554. doi: 10.1084/jem.20210554.

9. Bastard P, Rosen LB, Zhang Q et al. Autoantibodies against type I IFNs in patients with lifethreatening COVID-19. Science 2020;370(6515):eabd4585. doi: 10.1126/science.abd4585.

10. Williamson EJ, Walker AJ, Bhaskaran K, et al. Factors associated with COVID-19related death using OpenSAFELY. Nature 2020;584(7821):430-436. doi: 10.1038/ s41586-020-2521-4.

11. Stockmaier S, Stroeymeyt N, Shattuck EC, Hawley DM, Meyers LA, Bolnick DI. Infectious diseases and social distancing in nature. Science 2021;371(6533):eabc8881. doi: $10.1126 /$ science.abc 8881

12. Mathur R, Rentsch CT, Morton CE, et al. Ethnic differences in SARS-CoV-2 infection and COVID-19-related hospitalisation, intensive care unit admission, and death in 17 million adults in England: an observational cohort study using the OpenSAFELY platform. Lance 2021;397(10286):1711-1724. doi: 10.1016/S0140-6736(21)00634-6. 


\title{
Neurology ${ }^{\oplus}$ \\ Neuroimmunology \& Neuroinflammation
}

\author{
COVID-19 Severity in Multiple Sclerosis: Putting Data Into Context \\ Maria Pia Sormani, Irene Schiavetti, Luca Carmisciano, et al. \\ Neurol Neuroimmunol Neuroinflamm 2022;9; \\ DOI 10.1212/NXI.0000000000001105
}

This information is current as of November 9, 2021

Neurol Neuroimmunol Neuroinflamm is an official journal of the American Academy of Neurology.

Published since April 2014, it is an open-access, online-only, continuous publication journal. Copyright

Copyright $\left({ }^{\circ} 2021\right.$ The Author(s). Published by Wolters Kluwer Health, Inc. on behalf of the American

Academy of Neurology.. All rights reserved. Online ISSN: 2332-7812.

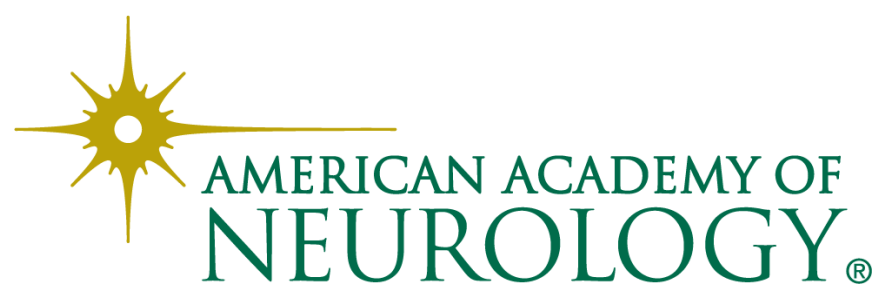




\section{Updated Information \& Services}

References

Citations

Subspecialty Collections

\section{Permissions \& Licensing}

Reprints including high resolution figures, can be found at: http://nn.neurology.org/content/9/1/e1105.full.html

This article cites 12 articles, 2 of which you can access for free at: http://nn.neurology.org/content/9/1/e1105.full.html\#\#ref-list-1

This article has been cited by 2 HighWire-hosted articles: http://nn.neurology.org/content/9/1/e1105.full.html\#\#otherarticles

This article, along with others on similar topics, appears in the following collection(s):

\section{COVID-19}

http://nn.neurology.org//cgi/collection/covid_19

Incidence studies

http://nn.neurology.org//cgi/collection/incidence_studies

Multiple sclerosis

http://nn.neurology.org//cgi/collection/multiple_sclerosis

Risk factors in epidemiology

http://nn.neurology.org//cgi/collection/risk_factors_in_epidemiology

Information about reproducing this article in parts (figures,tables) or in its entirety can be found online at:

http://nn.neurology.org/misc/about.xhtml\#permissions

Information about ordering reprints can be found online:

http://nn.neurology.org/misc/addir.xhtml\#reprintsus

Neurol Neuroimmunol Neuroinflamm is an official journal of the American Academy of Neurology.

Published since April 2014, it is an open-access, online-only, continuous publication journal. Copyright

Copyright $\odot 2021$ The Author(s). Published by Wolters Kluwer Health, Inc. on behalf of the American

Academy of Neurology.. All rights reserved. Online ISSN: 2332-7812.

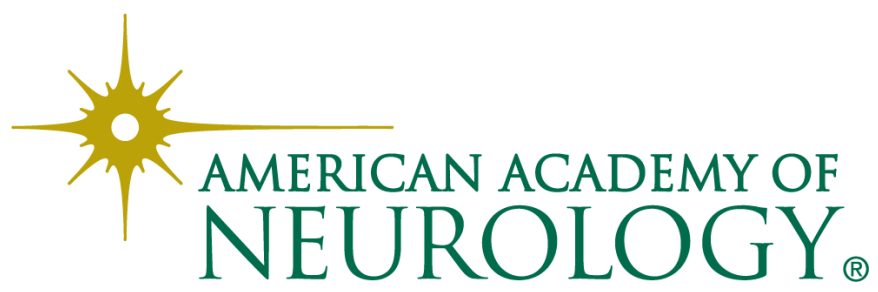

